Mathématiques et sciences humaines
Mathematics and social sciences

188 | Hiver 2009

Varia

\title{
Lorsque l'utilité, la gêne ou le confort sont recueillis sur une échelle de catégories : l'interaction dans le contexte multivarié
}

When the utility, the annoyance or the comfort are recorded with ordered categories, the interaction in the multivariate context, Math. Sci. hum. / Mathematics and Social Sciences, $n^{\circ}$ 188, 2009(4), p. 5-39.

Michel Maurin

\section{(2) OpenEdition} Journals

Édition électronique

URL : http://journals.openedition.org/msh/11194

DOI : $10.4000 / \mathrm{msh} .11194$

ISSN : 1950-6821

Éditeur

Centre d'analyse et de mathématique sociales de l'EHESS

Édition imprimée

Date de publication : 31 décembre 2009

Pagination : p. 5-39

ISSN : 0987-6936

\section{Référence électronique}

Michel Maurin, «Lorsque l'utilité, la gêne ou le confort sont recueillis sur une échelle de catégories l'interaction dans le contexte multivarié », Mathématiques et sciences humaines [En ligne], 188 | Hiver 2009, mis en ligne le 15 mars 2010, consulté le 23 juillet 2020. URL : http://journals.openedition.org/ msh/11194; DOI : https://doi.org/10.4000/msh.11194 


\title{
LORSQUE L’UTILITÉ, LA GÊNE OU LE CONFORT SONT RECUEILLIS SUR UNE ÉCHELLE DE CATÉGORIES : L'INTERACTION DANS LE CONTEXTE MULTIVARIÉ
}

\author{
Michel MAURIN ${ }^{1}$
}

RÉSUMÉ - Cet article concerne la numérisation d'une réponse catégorielle dans le cadre de la théorie du mesurage, comme peut l'être l'utilité en général, la gêne dans le cadre des nuisances, le confort en ergonomie. La méthode, à savoir les intervalles successifs d'Adams et Messick, est bien rodée pour des réponses en fonction d'une situation décrite par une grandeur numérique univariée ; nous examinons ici son extension au cas multivarié, avec la possibilité d'expliciter l'interaction entre variables.

MOTS-CLÉS -Échelle de catégories, Interaction, Intervalles successifs, Mesurage conjoint, Théorie du mesurage, Utilité

SUMMARY - When the utility, the annoyance or the comfort are recorded with ordered categories, the interaction in the multivariate context

This note is related to ordered categorical responses coming from experimental subjects as we generally do with the "utility" in econometry, annoyance in environmental surveys, or discomfort in ergonomy. Here we are working in the measurement theory framework. The appropriate measurement method is to use the successive intervals of Adams and Messick, which are well suited for univariate conditions when responses are given in relation to one descriptive variable; here we examine the multivariate extension, the first formal properties and the possibility to explicit the interaction between variables.

KEYWORDS - Category scales, Conjoint measurement, Interaction, Measurement theory, Successive intervals, Utility

\section{INTRODUCTION}

Nous nous plaçons dans le cas où, confronté à une situation $\mathrm{s}_{\mathrm{i}}$ il est attendu de donner une valeur numérique à «l'utilité » de la situation, communément représentée par un réel $\mathrm{u}\left(\mathrm{s}_{\mathrm{i}}\right)$ moyennant une correspondance $\mathrm{u}($.$) entre l'ensemble des situations et la$ grandeur utilité. Avec des situations prises au sens large, il s'agit de l'attribution d'un nombre à des entités. On connaît des disciplines qui ont réussi leur numérisation (géométrie, physique, ...), et dans plusieurs autres on assiste de manière récurrente à l'intention d'y parvenir également [Maurin, 2003(b)]. C'est le cas notamment de l'économie avec l'utilité de biens dans une grandeur autre que monétaire [Beinat, 1995], de la psycho-métrie [Martin, 1997 ; Michell, 1990], de la psycho-physique avec le dessein d'origine d'établir une relation numérique entre la stimulation et la sensation

\footnotetext{
${ }^{1}$ Institut National de Recherche sur les Transports et leur Sécurité, INRETS-LTE, case 24, 69675 Bron cedex, michel.maurin@inrets.fr
} 
ressentie [Bonnet, 1986 ; Falmagne, 1985 ; Martin, 1997 ; Stevens, 1971], et dans nos exemples de la gêne ressentie à la suite de l'exposition au bruit ou en ergonomie du confort en fonction de la géométrie d'un poste de conduite. Certains ont situé ces développements entre 1'Art et la Science [Annett, 2002], une expression assez bien choisie déjà utilisée dans [Keeney, 1992] en économie et par [Beinat, 1995] sous une forme dissymétrique more an art than a science à propos de l'utilité2

En effet le plus souvent au cours des enquêtes l'utilité n'est pas directement saisie sous une forme numérique et il y a lieu de la numériser en premier la plupart du temps. Nous commençons par rappeler une technique de psychophysique qui est adaptée aux réponses recueillies à l'aide d'une échelle de catégories ordonnées. Elle a été initiée par Thurstone, et formalisée sous le nom de law of categorical judgment par Saffir [Torgerson, 1958] mais implicitement attribuée à Thurstone lui-même [Debaty, 1967]. Par la suite elle a été reprise par la démarche de la théorie du mesurage (measurement theory) sour le nom des «intervalles successifs » (successive intervals) d'Adams et Messick [Suppes, Zinnes, 1963]. Dans le domaine des transports [Maurin, 2003(a), 2003(b)] ces questions se posent souvent pour la gêne acoustique ou pour le confort dans un poste de conduite. La numérisation sert à établir des relations dites d'exposition/réponse, ou lois du confort, en fonction de grandeurs physiques, et ce sont plus généralement des préoccupations analogues à celles de tout domaine relevant d'une recherche de l'utilité qui utilise des catégories ordonnées pour la réponse, en laissant bien entendu l'interprétation thématique au spécialiste du domaine en question.

L'objet de cet article est de montrer comment la méthode des intervalles successifs est applicable à une situation multidimensionnelle et peut conduire simplement à une méthode de mesurage analogue et concurrente au mesurage conjoint [Roberts, 1969 ; Michell, 1990 ; Bouyssou, Pirlot, 2002]. Pour cela nous présentons l'extension des intervalles successifs au cas de plusieurs variables, ses premières propriétés, ainsi que la possibilité d'expliciter l'interaction qui se manifeste entre les variables dans la réponse conjointe globale.

\section{UN RAPPEL DES INTERVALLES SUCCESSIFS DANS LE PARADIGME UNIDIMENSIONNEL}

Dans un premier temps, la réponse dépend de situations repérées par une seule variable.

\subsection{LE TABLEAU DE CONTINGENCE DES DONNÉES}

Les situations sont notées $\mathrm{s}_{\mathrm{i}}, \mathrm{i}=1 \ldots \mathrm{I}$ et pour chacune d'elles les sujets répondent à l'aide d'une échelle de catégories $C_{j}, j=1$...J. Les données qui en résultent peuvent être mises sous la forme d'un tableau de contingence à I lignes et $\mathrm{J}$ colonnes avec les effectifs $\mathrm{n}_{\mathrm{ij}}$ de répondants soumis à $s_{i}$ et ayant choisi $C_{j}$. On en déduit les distributions marginales $n_{i+}$ et $\mathrm{n}_{+\mathrm{j}}$ ainsi que les distributions conditionnelles des réponses avec les fréquences conditionnelles observées $\mathrm{f}_{\mathrm{jli}}=\mathrm{n}_{\mathrm{ij}} / \mathrm{n}_{\mathrm{i}+}$, (cf. Tableau 1$)$.

\footnotetext{
${ }^{2}$ Il y a aussi Raiffa en 1982 à propos de la négociation, sans oublier le Discours sur les sciences et les arts de Jean-Jacques Rousseau à l'Académie de Dijon en 1750 ; mais en l'occurrence les arts et les sciences étaient plutôt associés qu'opposés.
} 


\begin{tabular}{c|c|c|c|c|c|c|c} 
& $\mathrm{C}_{1}$ & $\mathrm{C}_{2}$ & & $\mathrm{C}_{\mathrm{j}}$ & & $\mathrm{C}_{\mathrm{J}}$ & \\
\hline $\mathrm{s}_{1}$ & & & & & & & \\
\hline & & & & & & & \\
\hline $\mathrm{s}_{\mathrm{i}}$ & & & & $\mathrm{n}_{\mathrm{ij}}$ & & & $\mathrm{n}_{\mathrm{i}}$ \\
\hline & & & & & & & \\
\hline $\mathrm{s}_{\mathrm{I}}$ & & & & & & & \\
\hline & & & & $\mathrm{n}_{+\mathrm{j}}$ & & &
\end{tabular}

TABLEAU 1. Le tableau de contingence des réponses en $\left\{\mathrm{C}_{\mathrm{j}}, \mathrm{s}_{\mathrm{i}}\right\}$

\subsection{LA LOI DES JUGEMENTS CATÉGORIELS}

Ces données sont le matériau de base de la «loi des jugements catégoriels » en psychophysique. Cette discipline cherche à établir une correspondance entre la variable de situation et la réponse numérisée, et dans ce cadre, l'une des avancées de Thurstone consiste à modéliser le processus de discrimination qui provoque une réponse (discriminal process) par une variable aléatoire sur $\mathrm{R}$ qui rend compte de la variablilité de la réponse, ... we can think of a frequency distribution on the psychological continuum of discriminal processes associated with that stimulus [Torgerson, 1958]. Dans une première loi dite « des jugements comparatifs » la préférence de $s_{i 1}$ sur $s_{i 2}$ est modélisée avec des variables aléatoires $\mathbf{U}_{\mathrm{i}}$ sur $\mathrm{R}$ et par la probabilité $\mathrm{P}\left(\mathbf{U}_{\mathrm{i} 2} \leq \mathbf{U}_{\mathrm{i} 1}\right)$.

La loi des jugements catégoriels en est une adaptation qui introduit d'autres variables aléatoires $\mathbf{T}_{\mathrm{j}}$ pour des stimulations de référence servant à la délimitation des catégories $\mathrm{C}_{\mathrm{j}}$. Dans ces conditions le choix de $\mathrm{C}_{\mathrm{j}}$ est modélisé par la probabilité $\mathrm{P}\left\{\right.$ réponse $\left.\in \mathrm{C}_{\mathrm{j}} \mathrm{s}_{\mathrm{i}}\right\}=\mathrm{P}\left(\mathbf{T}_{\mathrm{j}-1} \leq \mathbf{U}_{\mathrm{i}} \leq \mathbf{T}_{\mathrm{j}}\right)$, avec la probabilité cumulée $\mathrm{P}$ \{réponse $\in \cup_{\mathrm{k}=1 \ldots \mathrm{j}}$ $\left.\mathrm{C}_{\mathrm{k}} \mid \mathrm{s}_{\mathrm{i}}\right\}=\mathrm{P}\left(\mathbf{U}_{\mathrm{i}} \leq \mathbf{T}_{\mathrm{j}}\right)$ que l'on note encore ici $\mathrm{p}_{\mathrm{jli}}$. Dans le modèle on doit aussi se donner les lois de $\mathbf{U}_{\mathrm{i}}$ et $\mathbf{T}_{\mathrm{j}}$ pour calculer ces probabilités, et ce sont dans la quasi-totalité des applications des lois normales [Bonnet, 1986 ; Debaty, 1967 ; Torgerson, 1958]. Naturellement les espérances de ces lois $\mathrm{u}_{\mathrm{i}}=\mathrm{E}\left(\mathbf{U}_{\mathrm{i}}\right)$ et $\mathrm{t}_{\mathrm{j}}=\mathrm{E}\left(\mathbf{T}_{\mathrm{j}}\right)$ servent à coder les situations et les bornes d'intervalle, complétées par les bornes extrémales $\mathrm{t}_{0}=-\infty$ pour $\mathrm{C}_{1}$ et $\mathrm{t}_{\mathrm{J}}=\infty$ pour $\mathrm{C}_{\mathrm{J}}$.

La loi des jugements catégoriels consiste à identifier les inconnues $u_{i}$ et $t_{j}$ à partir de l'ajustement entre les $\mathrm{P}\left(\mathbf{U}_{\mathrm{i}} \leq \mathbf{T}_{\mathrm{j}}\right)$ du modèle et les fréquences cumulées recueillies $\sum_{\mathrm{k}=1 \ldots \mathrm{j}} \mathrm{f}_{\mathrm{kli}}$, avec plusieurs options éventuelles selon diverses sous-hypothèses que l'on fait à propos des variances et des covariances entre les $\mathbf{U}_{\mathrm{i}}$ et $\mathbf{T}_{\mathrm{j}}$ [Bonnet, 1986 ; Torgerson, 1958]. On note à ce propos que Thurstone utilisait indifféremment les deux termes de loi et de méthode, et que l'on pourrait utiliser l'expression de loi-méthode [Martin, 1997]. 


\subsection{DES JUGEMENTS CATÉGORIELS AUX « INTERVALLES SUCCESSIFS »}

\subsubsection{La théorie du mesurage (measurement theory)}

La théorie du mesurage est plus générale. Elle s'occupe de la numérisation des entités pré-quantitatives (numerical assignment), et elle n'envisage de représentation numérique de ces entités qu'après avoir démontré leur existence [Suppes, Zinnes, 1963 ; Pfanzagl, 1971 ; Roberts, 1979]. Ordinairement les observations qualitatives appartiennent à des ensembles qui présentent malgré tout certaines structures ensemblistes (pré-ordres, lois de compositions internes, ...), et en premier la théorie s'applique à établir qu'il existe bien un homomorphisme entre cet ensemble structuré et un espace d'arrivée numérique, $\mathrm{R}$ ou $\mathrm{R}^{\mathrm{n}}$, (un théorème de Représentation). Lorsqu'une telle représentation existe, il est courant qu'elle ne soit pas unique, et il s'agit ensuite de caractériser l'ensemble des solutions à l'aide d'un théorème de Caractérisation (Uniqueness). C'est à ce propos que l'on évoque les types d'échelle de mesurage comme les échelles absolues, de rapport, d'intervalle, ordinales et nominales par exemple, puis que l'on examine la pertinence (meaningfulness) des opérations numériques que l'on peut faire sur les valeurs obtenues. Cela s'oppose ainsi délibérément à toute sorte de numérisation posée a priori, et cela malgré un quantitative impérative fortement répandu [Michell, 1990]. Et puisque la théorie repose sur l'explicitation de relations observées sous forme d'axiomes ou de conditions de mesurage, puis sur des théorèmes qui prennent ces axiomes comme hypothèses, la démarche du mesurage permet de «révéler» la quantification des données qui est « contenue » dans des informations simplement qualitatives au départ.

\subsubsection{La rénovation d'Adams et Messick}

Ces deux auteurs ont repris le schéma thurstonien des réponses catégorielles selon le point de vue du mesurage. Dans un premier temps, ils ont retenu une autre option pour les lois de probabilité, les $\mathbf{U}_{\mathrm{i}}$ ont pour fonction de répartition $\mathrm{F}_{\mathrm{i}}(\mathrm{x})=\mathrm{F}\left(\left(\mathrm{x}-\mathrm{u}_{\mathrm{i}}\right) / \sigma_{\mathrm{i}}\right)$ avec une fonction de répartition commune $F$ définie sur $R$, normale ou pas, les $\mathbf{T}_{j}$ suivent des distributions de Dirac en $\mathrm{t}_{\mathrm{j}}$, et ces variables sont mutuellement indépendantes. Il en résulte que la probabilité $\mathrm{p}_{\mathrm{jli}}$ de l'événement $\left(\mathbf{U}_{\mathrm{i}} \leq \mathbf{T}_{\mathrm{j}}\right)$ est ici égale à $\mathrm{F}\left(\left(\mathrm{t}_{\mathrm{j}}-\mathrm{u}_{\mathrm{i}}\right) / \sigma_{\mathrm{i}}\right)$, et que l'on peut écrire encore $\mathrm{z}_{\mathrm{ij}}=\left(\mathrm{t}_{\mathrm{j}}-\mathrm{u}_{\mathrm{i}}\right) / \sigma_{\mathrm{i}}, \mathrm{i}=1 \ldots \mathrm{I}, \mathrm{j}=1 \ldots \mathrm{J}-1$ en posant $\mathrm{z}_{\mathrm{ij}}=\mathrm{F}^{-1}\left(\mathrm{p}_{\mathrm{jli}}\right)$.

Adams et Messick ont ensuite remarqué que les $z_{i j}$ vérifient un système de relations algébriquement équivalentes de la forme

$$
\mathrm{z}_{\mathrm{kj}}=\alpha_{\mathrm{kl}} \mathrm{z}_{\mathrm{lj}}+\beta_{\mathrm{kl}} \quad\left[\mathrm{C}_{\mathrm{AM}}\right]
$$

avec des coefficients $\alpha_{\mathrm{kl}}$ positifs pour tout couple d'indices de lignes $1, \mathrm{k}=1, \ldots$, I et toute colonne $\mathrm{j}$. Ces nouvelles relations dans lesquelles ne figurent plus les représentations $\mathrm{u}\left(\mathrm{s}_{\mathrm{i}}\right)=\mathrm{u}_{\mathrm{i}}$ et $\mathrm{t}\left(\mathrm{C}_{\mathrm{j}}\right)=\mathrm{t}_{\mathrm{j}} \mathrm{s}$ 'expriment ainsi de manière intrinsèque avec les seuls éléments $\mathrm{p}_{\mathrm{jli}}$ ou $\mathrm{z}_{\mathrm{ij}} \mathrm{du}$ tableau. Elles signifient que l'on passe de toute ligne d'indice $\mathrm{k}$ à toute autre ligne 1 des $z_{\mathrm{ij}}$ par une transformation affine qui ne dépend que des indices $\mathrm{k}$ et 1 ; elles sont directement vérifiables au vu des données, et elles constituent les axiomes ou les conditions qu'Adams et Messick ont retenus pour le mesurage des réponses par échelles de catégories ordonnées. 
En dernier, on peut avec ces axiomes démontrer un théorème de représentation vis-à-vis de l'existence des $\mathrm{u}_{\mathrm{i}}$ et $\mathrm{t}_{\mathrm{j}}$, ainsi qu'un théorème de caractérisation qui précise que le mesurage obtenu est fait avec une échelle d'intervalle, la même échelle pour les $\mathrm{u}_{\mathrm{i}}$ et les $\mathrm{t}_{\mathrm{j}}$ [Adams, Messick, 1958 ; Suppes, Zinnes, 1963 ; Maurin, 1986]. C'est cette reformulation des jugements catégoriels qui constitue la méthode des intervalles successifs, Figure 1, et bien entendu on conserve avec elle l'élargissement de la loiméthode de Thurstone à plusieurs sortes de recueils, des opinions, des attitudes, des valeurs et autres stimuli susceptibles d'être comparés [Martin, 1997].

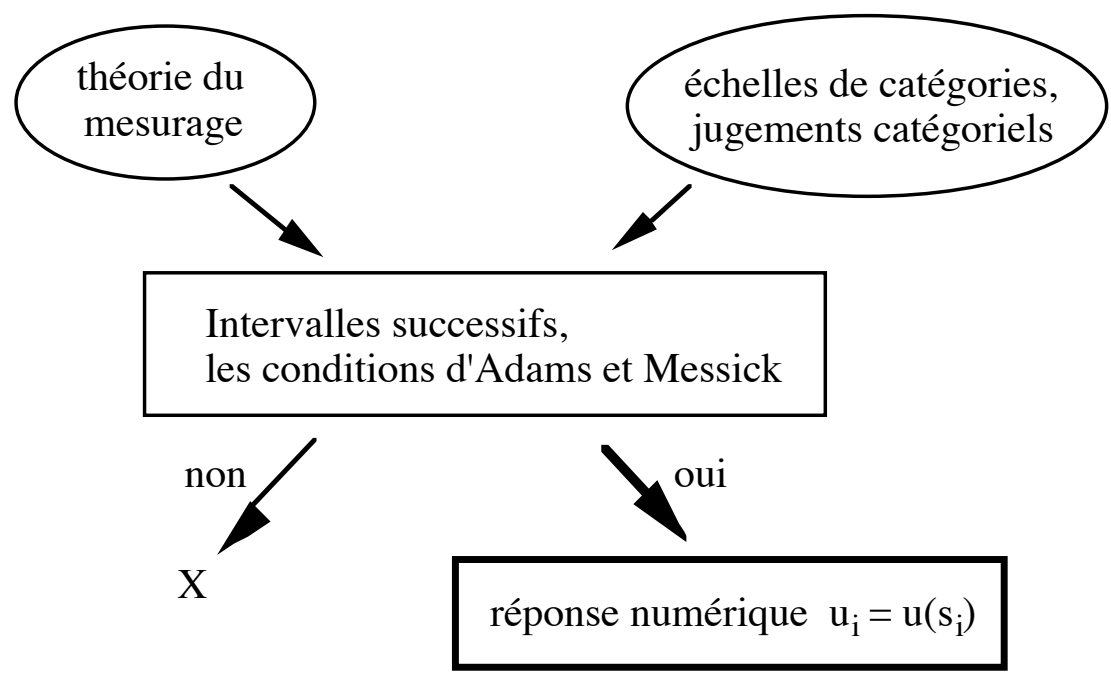

FIGURE 1. Les intervalles successifs d'Adams et Messick

\subsection{LE BRUIT DES DONNÉES ET LE CALCUL DES VALEURS NUMÉRIQUES}

Cependant les données observées sont des effectifs $n_{i j}$, des fréquences $f_{j l i}$ et des fréquences cumulées $q_{j l i}=\sum_{k=1 \ldots j} f_{k l i}$ qui sont des estimateurs des probabilités $p_{j l i}$ des conditions. C'est avec ces données expérimentales que l'on doit expertiser les conditions $\left[\mathrm{C}_{\mathrm{AM}}\right]$ ou les relations équivalentes $\mathrm{z}_{\mathrm{ij}}^{\mathrm{e}}=\left(\mathrm{t}_{\mathrm{j}}-\mathrm{u}_{\mathrm{i}}\right) / \sigma_{\mathrm{i}}=\mathrm{a}_{\mathrm{i}} \mathrm{t}_{\mathrm{j}}+\mathrm{b}_{\mathrm{i}}$ en posant $\mathrm{z}_{\mathrm{ij}} \mathrm{e}=\mathrm{F}^{-1}\left(\mathrm{q}_{\mathrm{ji}}\right)$, $a_{i}=1 / \sigma_{i}$ et $b_{i}=-u_{i} / \sigma_{i}$, moyennant le choix concret d'une fonction de répartition $F$.

Il faut donc appliquer une technique de mesurage probabiliste [Falmagne, 1976]. Sur le plan théorique on développe un test d'hypothèse pour accepter l'hypothèse d'une représentation [Hamerle, Tutz, 1980 ; Maurin, 1986], et sur le plan pratique, quand l'hypothèse est acceptée, les équations $\mathrm{z}_{\mathrm{ij}} \mathrm{e}=\mathrm{a}_{\mathrm{i}} \mathrm{t}_{\mathrm{j}}+\mathrm{b}_{\mathrm{i}}$ forment un système à $2 \mathrm{I}+\mathrm{J}-1$ inconnues $a_{i}, b_{i}, t_{j}$, et à $I(J-1)$ relations auxquelles il faut ajouter deux conditions supplémentaires pour fixer les coefficients de l'échelle d'intervalle commune. Ce système est surdéterminé dès que (I-1) (J-3) est positif, et il ne peut directement se prêter à une résolution numérique. Pour y remédier, on pose l'écart quadratique ou stress $\mathrm{Q}_{\mathrm{F}}=\sum_{\mathrm{ij}}\left\{\mathrm{z}_{\mathrm{ij}} \mathrm{e}_{\mathrm{i}} \mathrm{a}_{\mathrm{j}}-\mathrm{b}_{\mathrm{i}}\right\}^{2}$ qui est identiquement nul sous les conditions [ $\mathrm{C}_{\mathrm{AM}}$ ] pour les $z_{i j}{ }_{i j}$, et l'on recherche les valeurs de $a_{i}$, $b_{i}$ et $t_{j}$ pour lesquelles $Q_{F}$ est minimal, comme on le fait déjà pour la loi des jugements catégoriels [Maurin, 2003(a), 2003(b)]. Dans le même temps on peut envisager plusieurs fonctions $F$ et retenir celle qui conduit au minimum le plus faible. 
Pour les applications, nous avons retenu les lois classiques normale et logistique, la loi des extrêmes de Fréchet Fisher Tippett et la loi FFT changée de signe de fonction de répartition $\mathrm{F}(\mathrm{x})=1-\exp \left(-\mathrm{e}^{\mathrm{x}}\right)$. Les deux dernières sont plus adaptées pour des données qui manifestent de l'asymétrie dans leur distribution.

\subsection{AUTRES INFORMATIONS NUMÉRIQUES}

L'optimisation est dans le même temps un algorithme constructif pour les valeurs numériques inconnues que l'on recherche. En dernier lieu des considérations distributionnelles classiques permettent de définir une valeur ponctuelle $c_{j}=c\left(C_{j}\right)$ pour chaque catégorie, comme une sorte de résumé barycentrique (Annexe 1), ainsi que des pseudo-bornes extrémales pour $t_{0}$ et $t_{J}$ avec des quantiles (Annexe 2). Ces résultats sont dus aux conséquences naturelles des modèles qui utilisent les distributions de probabilité, mais ils n'interviennent pas dans les conditions de mesurage. Par conséquent les $c_{j}$ ne sont pas des numérisations ex abrupto comme dans une mise en oeuvre de scaling ou de scoring, et ils ne contournent pas les conditions du mesurage. Ce sont ainsi des valeurs numériques bien plus pertinentes que le rang des catégories souvent prises comme codage forfaitaire [Maurin, 2003(b), 2006] et jugées a bit hair raising [Luce, Galanter, 1963] avec un certain euphémisme. En outre, l'échelle d'intervalle des $u_{i}$ et $t_{j}$ doit être calibrée en pratique avec deux conditions, cela peut être $\mathrm{u}_{1}=1$ et $\mathrm{u}_{\mathrm{I}}=\mathrm{I}$, mais l'option $\mathrm{c}_{1}=1$ et $\mathrm{c}_{\mathrm{J}}=\mathrm{J}$ se révèle commode.

\subsection{DES ENSEIGNEMENTS DU MESURAGE ET DES INTERVALLES SUCCESSIFS}

\subsubsection{Measurement, mesurage et mesure}

D'une manière générale, il est patent que le «nombre » figure dans de nombreuses (!) activités, que son usage est ubiquitous comme le dit Hand [2004] d'emblée, et qu'il exerce une attirance notable. Parmi les avantages qu'on lui accorde le nombre permet de tenir un discours qui va au-delà de l'expression verbale et qualitative, il peut guider vers des conséquences inaccessibles au raisonnement verbal [Boudon, 1967], ou encore ... the practical advantage of taking numbers instead of words is unquestionnable [Pfanzagl, 1971].

C'est notamment pour disposer de nombres et dans le même temps pour se prémunir contre des excès possibles que la théorie du mesurage met l'accent sur les précautions à prendre avant toute chose. L'attention portée sur le respect des relations ensemblistes pré-quantitatives observées sur les données constitue ainsi un bon garant de la qualité vis-à-vis de la représentativité et de la signifiance des nombres. Avec eux on ne peut qu'être mieux en mesure (!) de tenir un discours numérique satisfaisant.

En faisant ainsi on note que cette théorie va au-delà des mesures auxquelles on est le plus habitué. En effet, les mesures ordinaires sur des grandeurs en géométrie (longueur, aire, ...), en physique (masse, durée, ...) ou en économie (valeur monétaire, volume de production, ...) sont le plus souvent faites avec des échelles de rapport dont le zéro a un sens ; par ailleurs, la mesure provient d'un appareil comme un mètre, un voltmètre, .... Avec le mesurage au contraire les nombres sont obtenus moyennant des représentations qui vérifient des conditions de conformité avec les données. À ce titre, la théorie est avant tout une théorie de l'assignation numérique pertinente et des opérations admissibles sur les valeurs acquises, et l'on doit rappeler le rôle précurseur de Stevens dans cette formulation [Martin, 1997]. Un autre facteur d'élargissement 
provient du fait que l'on y rencontre plusieurs types d'échelles de mesure de manière plus explicite qu'ordinairement. C'est le cas notamment de l'échelle d'intervalle avec ses règles de discours un peu différentes; les zéros des températures Celsius ou Fahrenheit ne sont que des repères contingents, et ce sont les différences ou écarts de température dont les rapports ont un sens. Incidemment il y a aussi des précautions à prendre au niveau du langage à cause de la mesure en mathématiques, en matière comportementale, en musique, voire de la métrique en poésie.

\subsubsection{Intervalles successifs}

Les intervalles successifs sont une mise en oeuvre du mesurage et l'on obtient simultanément une mesure $\mathrm{u}_{\mathrm{i}}$ ou $\mathrm{u}\left(\mathrm{s}_{\mathrm{i}}\right)$ des stimuli et $\mathrm{t}_{\mathrm{j}}$ des bornes de catégorie. Parmi les enseignements numériques que l'on en retire, on a les étendues respectives $\Delta \mathrm{t}_{\mathrm{j}}=\mathrm{t}_{\mathrm{j}}-\mathrm{t}_{\mathrm{j}-1}$ des intervalles correspondant aux catégories de l'échelle de départ, à savoir leur amplitude subjective respective, ainsi que les espacements $\Delta \mathrm{c}_{\mathrm{j}}=\mathrm{c}_{\mathrm{j}}-\mathrm{c}_{\mathrm{j}-1}$ entre les barycentres ponctuels associés des catégories. Simultanément les $\mathrm{u}_{\mathrm{i}}$ qui mesurent la réponse à chaque $s_{i}$ permettent de voir où se situent ces stimulations par rapport aux catégories de l'échelle.

En second lieu les couples $\left\{u_{i}, s_{i}\right\}$, ou encore les couples numériques $\left\{u_{i}, x_{i}\right\}$, lorsque les $\mathrm{x}_{\mathrm{i}}$ sont les intensités de la grandeur physique qui crée la stimulation, établissent une correspondance entre une stimulation physique $\mathrm{S}$ et la réponse numérisée U, c'est-à-dire souscrivent au dessein psychophysique d'origine. Il s'agit d'une relation de type générique dose/réponse, exposition/réponse, dite encore loi d'utilité, d'impact, de confort, pour toute exposition qui entre dans le champ des «stimuli thurstoniens ».

En dernier, les $\mathrm{u}_{\mathrm{i}}$ étant sur une échelle d'intervalle, on en déduit immédiatement les stimulations qui correspondent aux réponses extrémales. Et lorsque l'on met la correspondance sous la forme d'un graphe dans un repère avec $x$ en abscisse et $u$ en ordonnée, on observe ses plages de monotonie croissante ou décroissante avec ses maxima ou minima. En outre avec les accroissements $\Delta \mathrm{u}_{\mathrm{i}}=\mathrm{u}_{\mathrm{i}}-\mathrm{u}_{\mathrm{i}-1}$ et $\Delta \mathrm{x}_{\mathrm{i}}=\mathrm{x}_{\mathrm{i}}-\mathrm{x}_{\mathrm{i}-1}$ (tous deux sur échelle de rapport) les pentes par intervalles $\theta_{\mathrm{i}}=\Delta \mathrm{u}_{\mathrm{i}} / \Delta \mathrm{x}_{\mathrm{i}}$ permettent d'observer les changements de monotonie et donc la convexité par morceaux, ainsi que la présence éventuelle de points d'inflexion dans la loi de réponse, ( $S$-shaped function, ogive curve). On a aussi directement les rapports entre eux des divers accroissements $\Delta \mathrm{u}_{\mathrm{i}}$ ou des pentes $\theta_{\mathrm{i}}$.

Sur un plan plus historique et méthodologique les intervalles successifs font écho au premier problème que s'était posé Thurstone avant même les jugements comparatifs, à savoir la construction d'échelles catégorielles comprenant des intervalles « apparemment égaux» en recherchant des marqueurs sémantiques [Bonnet, 1986 ; Debaty, 1967]. De ce fait les intervalles successifs rendent plus ou moins obsolètes ces premiers travaux sur les recueils catégoriels. 


\section{DES EXEMPLES UNIVARIÉS}

\subsection{LA GÊNE DU BRUIT ROUTIER}

Le premier exemple concerne une étude sur le bruit de la circulation routière et la gêne globale qui en résulte. La réponse est recueillie avec une échelle comprenant 4 catégories et la situation où l'exposition sonore est mesurée, par un indice de bruit équivalent Leq de $8 \mathrm{~h}$ à 20h, l'indice acoustique réglementaire en 1986 (les niveaux sont regroupés par plages de 3 décibels d'étendue à l'exception de plages plus larges aux extrémités) [Maurin, Lambert et Alauzet, 1988 ; Maurin, 2003(b)].

Les données forment un tableau de contingence à 4 colonnes et 7 lignes, il contient quelques cases vides qui excluent le respect strict des conditions d'Adams et Messick, cependant le calcul s'en accommode dans le cadre d'un mesurage avec une étape d'optimisation de stress, et les données ne sont pas trop distantes du respect des $\left[\mathrm{C}_{\mathrm{AM}}\right]$. Le minimum du stress est ici obtenu avec la loi de Fréchet Fisher Tippett changée de signe.

\begin{tabular}{c|cccc}
$\mathrm{L}_{\mathrm{i}}, \mathrm{dB}$ & \multicolumn{5}{c}{$\mathrm{C}_{\mathrm{j}}, \mathrm{j}=1 \ldots 4$} \\
50,0 & 72 & 7 & 1 & 0 \\
57,0 & 46 & 8 & 2 & 0 \\
60,0 & 48 & 8 & 7 & 3 \\
63,0 & 36 & 10 & 9 & 2 \\
66,0 & 24 & 12 & 8 & 6 \\
69,0 & 15 & 10 & 4 & 4 \\
73,5 & 15 & 6 & 7 & 5
\end{tabular}

TABLEAU 2. Données de l'enquête de gêne diurne de 1986

On obtient ainsi les $\mathrm{u}\left(\mathrm{s}_{\mathrm{i}}\right)$

$$
\begin{array}{lllllll}
1,634 & 1,942 & 2,136 & 2,525 & 2,914 & 2,917 & 3,061
\end{array}
$$

les bornes d'intervalle $t_{j}$ et les quantiles extrémaux (en italique), (cf. Figure 2),

$$
\begin{array}{lllll}
-3,011 & 2,480 & 3,132 & 3,734 & 4,349
\end{array}
$$

Toutes les stimulations sont situées dans les deux premiers intervalles, et les intervalles centraux $\mathrm{C}_{2}, \mathrm{C}_{3}$ ont des étendues comparables. On en retire aussi la correspondance numérique $\left\{\mathrm{L}_{\mathrm{i}} \equiv \mathrm{x}_{\mathrm{i}}, \mathrm{u}_{\mathrm{i}}\right\}$ entre la stimulation et la réponse de gêne numérisée (cf. Figure 3). La gêne croît en fonction du niveau de bruit avec une croissance qui s'accélère avant de manifester une saturation pour les niveaux de bruit élevés. C'est la correspondance entre stimulation et réponse qui a été retenue dans le cadre d'une approche multicritère de plusieurs nuisances bruit et pollution de l'air par l'intermédiaire de leurs « lois de réponse » [Rousval, 2005].

Pour montrer les ressources de la méthode nous l'avons appliquée sur un tableau analogue avec le niveau de bruit pendant la nuit et la réponse de gêne au réveil. Ici les conditions $\left[\mathrm{C}_{\mathrm{AM}}\right]$ sont très nettement violées et il n'y a aucune possibilité de valider la représentation numérique. Ces données sont ainsi un contre-exemple pour illustrer le fait que l'on ne peut appliquer les intervalles successifs à n'importe quel tableau [Maurin, 2003(a), 2003(b)] ; mais à cette occasion, on note que les garde-fous du mesurage rejoignent le bon sens puisque l'on ne peut guère au réveil répondre en conscience en fonction de l'exposition à laquelle on a été soumis durant son sommeil. 


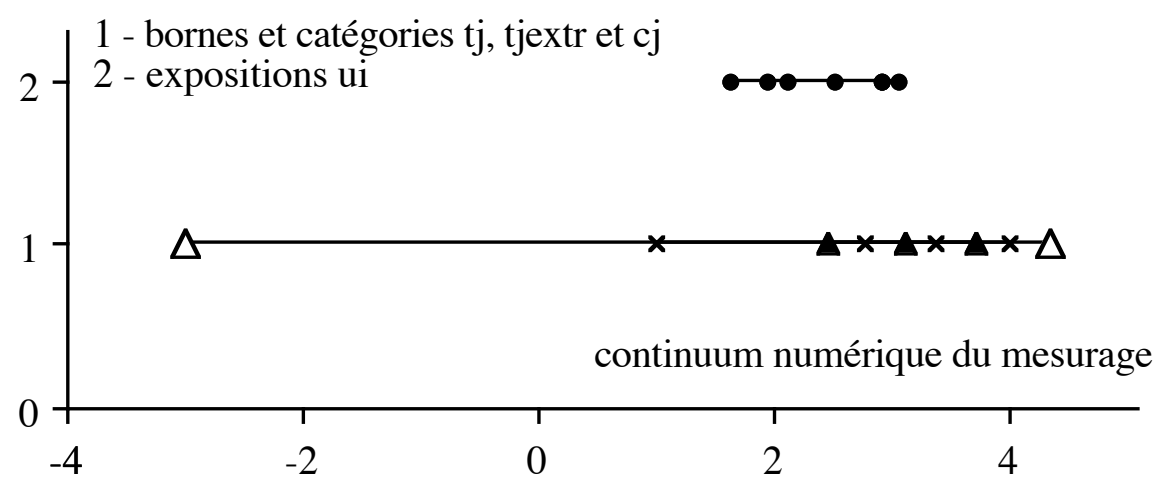

FIGURE 2. Les divers mesurages du Tableau 2

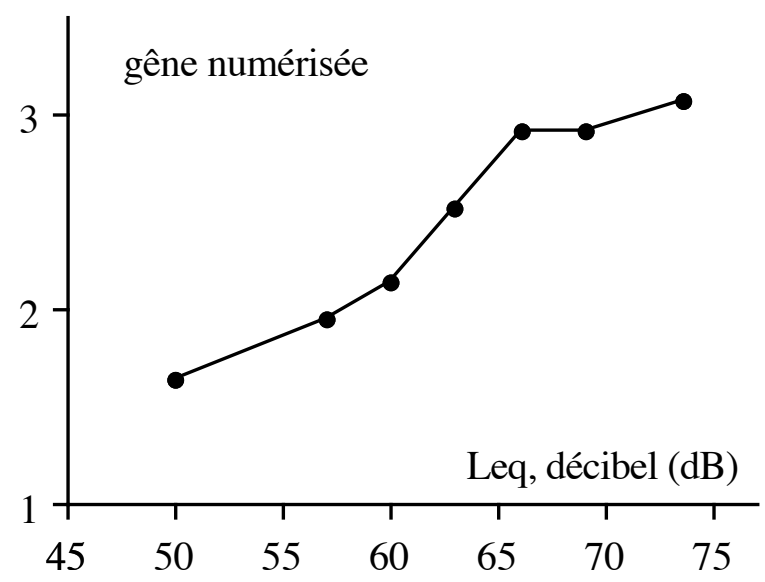

FIGURE 3. La correspondance niveau de bruit $\mathrm{L}_{\mathrm{i}}$ et gêne numérisée $\mathrm{u}_{\mathrm{i}}$, (Tableau 2)

\subsection{LE CONFORT DANS UN POSTE DE CONDUITE}

Le second exemple concerne une étude de gêne gestuelle en ergonomie à propos de l'atteinte d'une cible par un sujet assis à un poste de conduite (par la main droite). Les réponses sont recueillies par une échelle à 7 catégories et la cible est repérée par 3 variables géométriques, la hauteur, la distance et la position angulaire de la cible par rapport au sujet [Chevalot, Wang, 2004]. Les catégories extrémales étant assez peu représentées les catégories ont été ramenées à 5 en regroupant les catégories aux extrémités [Maurin, 2006].

L'exemple ci-dessous concerne l'épreuve en fonction de l'orientation de la cible par rapport au sujet, avec 5 positions de $-45^{\circ}$ à $+135^{\circ}$ par incrément de $45^{\circ}$.

\begin{tabular}{c|rcrrr}
$\mathrm{x}_{\mathrm{i}}$ en & \multicolumn{5}{c}{$\mathrm{C}_{\mathrm{j}}, \mathrm{j}=1 \ldots 5$} \\
-45 & 182 & 284 & 198 & 49 & 7 \\
0 & 288 & 276 & 154 & 40 & 2 \\
45 & 204 & 261 & 234 & 56 & 5 \\
90 & 99 & 289 & 264 & 78 & 30 \\
135 & 20 & 102 & 257 & 188 & 193
\end{tabular}

TABLEAU 3. Réponses de l'inconfort en fonction de l'orientation 
Le minimum du stress est obtenu avec la loi normale. Les catégories centrales $\mathrm{C}_{2}$, $\mathrm{C}_{3}, \mathrm{C}_{4}$ sont d'égales amplitudes (cf. Figure 4), et la correspondance $\left\{\mathrm{x}_{\mathrm{i}}, \mathrm{u}_{\mathrm{i}}\right\}$ affiche ici une allure parabolique avec une décroissance initiale, un minimum pour 0 degré et une forte croissance quand l'angle augmente (cf. Figure 5). Cette nouvelle forme montre la souplesse et le potentiel d'utilisation des intervalles successifs, une nouvelle fois en accord avec le bon sens.

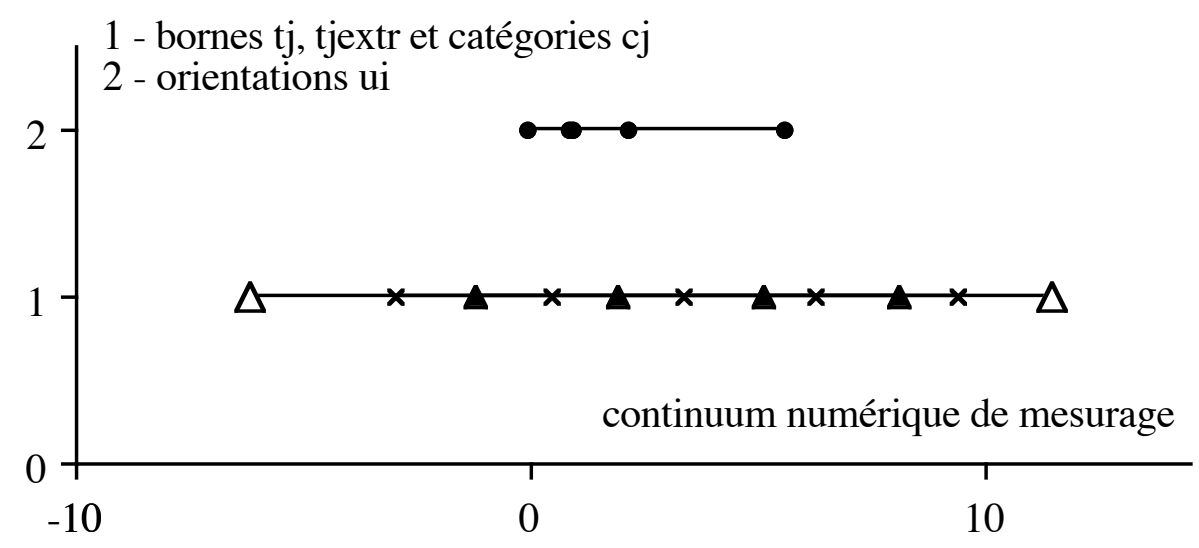

FIGURE 4. Les divers mesurages du Tableau 3

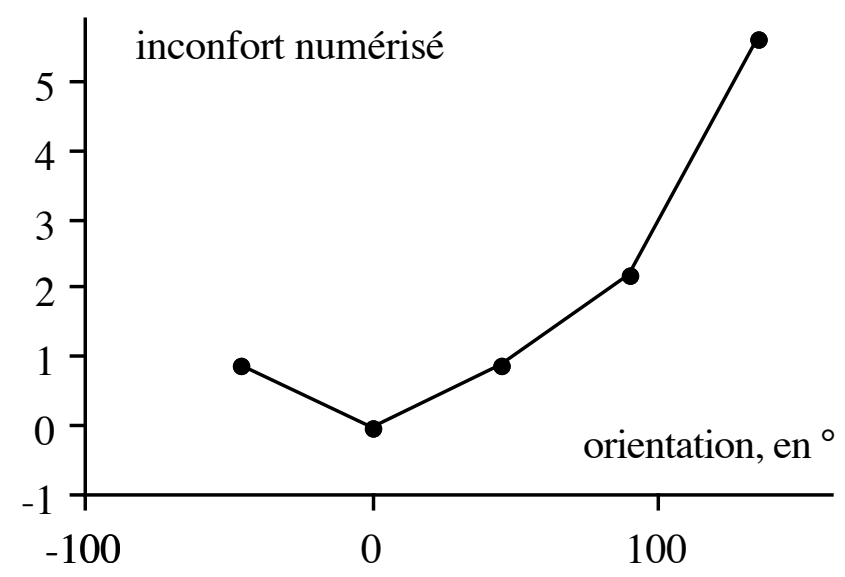

FIGURE 5. La correspondance orientation $\mathrm{x}_{\mathrm{i}}$ et inconfort numérisé $\mathrm{u}_{\mathrm{i}}$, (Tableau 3)

\section{LE CONTEXTE MULTIDIMENSIONNEL}

\subsection{UNE VISION ÉLARGIE DES DONNÉES}

Il n'y a rien dans ce qui précède qui se rapporte explicitement à l'ensemble des situations et à sa structure algébrique ; on peut donc envisager le cas où l'indice qui repère les situations est un indice composite $\{\mathrm{i}\}$ correspondant à plusieurs variables différentes unidimensionnelles $\mathrm{x}_{\mathrm{k}} \quad \mathrm{k}=1 \ldots \mathrm{K}$ prenant des valeurs $\mathrm{x}_{\mathrm{ki}_{\mathrm{k}}}$. Dans ce contexte multidimensionnel la situation est indexée par un K-uple et les effectifs de réponse $\mathrm{n}_{\{\mathrm{i}\} \mathrm{j}}$ recueillis figurent dans un pavé de contingence à $\mathrm{K}+1$ dimensions. 
Puisque l'on peut se ramener à un tableau à deux dimensions avec la collection des situations $\mathrm{s}_{\{\mathrm{i}\}}$ en lignes, on retrouve la disposition précédente pour expertiser sur ce nouveau tableau les axiomes $\left[\mathrm{C}_{\mathrm{AM}}\right]$ du mesurage. Comme précédemment lorsque cette vérification statistique est «satisfaisante » il en résulte comme première conséquence que l'on a la possibilité d'établir une correspondance numérique entre un K-uple de valeurs par jeux de situations et une réponse conjointe $\mathrm{u}_{\{\mathrm{i}\}}$ ou $\mathrm{u}\left(\mathrm{s}_{\{\mathrm{i}\}}\right)$ mesurée dans $\mathrm{R}$. C'est en l'occurrence une fonction à plusieurs variables pouvant encore être notée $\mathrm{u}_{\mathrm{i}_{1} \mathrm{i}_{2} \ldots \mathrm{i}_{\mathrm{K}}}$ ou $\mathrm{h}\left(\mathrm{s}_{1 \mathrm{i}_{1}}, \mathrm{~s}_{2 \mathrm{i}_{2}}, \ldots, \mathrm{s}_{\mathrm{Ki}_{\mathrm{K}}}\right)$ qui constitue la «surface de réponse » à l'issue de la numérisation par intervalles successifs. Cette surface est l'analogue multidimensionnel aux lois unidimensionnelles du paragraphe précédent, elle a les mêmes qualités de représentativité numérique, et l'on peut avec elle en déduire des nouvelles informations propres au contexte multidimensionnel. On en déduit notamment des coupes partielles univariées de la réponse conjointe en gardant K-1 arguments constants et en faisant varier le dernier.

\subsection{UNE REFORMULATION DES INTERVALLES SUCCESSIFS UNIVARIÉS}

Sur un autre plan, on peut énoncer une formulation plus probabiliste des intervalles successifs qu'en $\S 2$ en introduisant une nouvelle variable aléatoire $\mathbf{S}$ pour modéliser les différentes situations au cours de l'expérimentation avec les modalités $\mathrm{s}_{\mathrm{i}}$. Dans ces conditions la variable de réponse $\mathbf{U}_{\mathrm{i}}$ relative à chaque situation est une variable conditionnée $\mathbf{U I S}=\mathrm{s}_{\mathrm{i}}$, et les espérances $\mathrm{u}_{\mathrm{i}}=\mathrm{E}\left(\mathbf{U}_{\mathrm{i}}\right)$ deviennent des espérances conditionnelles $\mathrm{E}\left(\mathbf{U} \mid \mathbf{S}=\mathrm{s}_{\mathrm{i}}\right)$. Quant aux bornes, on a $\mathrm{t}_{\mathrm{j}}=\mathrm{E}\left(\mathbf{T}_{\mathrm{j}}\right)=\mathrm{E}\left(\mathbf{T}_{\mathrm{j}} \mathbf{S}=\mathrm{s}_{\mathrm{i}}\right)$ avec des distributions pour les $\mathbf{T}_{\mathrm{j}}$ indépendantes de celle de $\mathbf{S}$; bien entendu les $\mathrm{u}_{\{\mathrm{i}\}}$ et les $\mathrm{t}_{\mathrm{j}}$ demeurent sur une même échelle d'intervalle.

La loi de réponse peut ainsi être formulée par l'expression probabiliste $\mathrm{E}(\mathbf{U I S})$; c'est une espérance conditionnelle, et l'on peut rappeler ses principales propriétés :

- $\mathrm{E}(\mathbf{U} \mid \mathbf{S})$ est une transformée $\mathrm{f}(\mathbf{S})$ de la variable aléatoire $\mathbf{S}$,

- la relation de l'espérance totale : $\mathrm{E}(\mathbf{U})=\mathrm{E}_{\mathrm{S}}(\mathrm{E}(\mathbf{U} \mid \mathbf{S}))$,

- la relation de la variance totale : $\operatorname{var}(\mathbf{U})=\mathrm{E}_{\mathrm{S}}(\operatorname{var}(\mathbf{U I S}))+\operatorname{var}_{\mathrm{S}}(\mathrm{E}(\mathbf{U} \mid \mathbf{S}))$.

\subsection{LE PASSAGE AU MULTIVARIÉ}

\subsubsection{Un formalisme plus étendu}

Il en est de même dans le cas d'une exposition décrite par un indice multivarié comme en $\S 4.1$, et sous réserve des $\left[\mathrm{C}_{\mathrm{AM}}\right]$ correspondantes, les résultats des intervalles successifs peuvent être mis sous la formulation conditionnelle $\mathrm{u}_{\{\mathrm{i}\}}=\mathrm{E}\left(\mathbf{U} \mid \mathbf{S}=\mathrm{s}_{\{\mathrm{i}\}}\right)$ ou $\mathrm{E}\left(\mathbf{U} \mid \mathbf{S}_{1}=\mathrm{s}_{1 \mathrm{i}_{1}}, \mathbf{S}_{2}=\mathrm{s}_{2 \mathrm{i}_{2}}, \ldots, \mathbf{S}_{\mathrm{K}}=\mathrm{s}_{\mathrm{Ki}_{\mathrm{K}}}\right)$ moyennant les valeurs numériques $\mathrm{s}_{\mathrm{ki}_{\mathrm{k}}}$ des variables de conditionnement univariées. La forme générale $\mathrm{E}\left(\mathbf{U} \mid \mathbf{S}_{1}, \mathbf{S}_{2}, \ldots, \mathbf{S}_{\mathrm{K}}\right)$ montre la dépendance de la variable réponse $\mathbf{U}$ en fonction du K-uple des variables expositions $\left(\mathbf{S}_{1}, \mathbf{S}_{2}, \ldots, \mathbf{S}_{\mathrm{K}}\right)$.

Cette expression bénéficie à son tour des propriétés de l'espérance conditionnelle multiple. En effet en présence de deux variables de conditionnement $\mathbf{S}_{1}$ et $\mathbf{S}_{2}$ pour simplifier, on a les relations $\mathrm{E}_{\mathrm{S} 2}\left(\mathrm{E}\left(\mathbf{U} \mid \mathbf{S}_{1}, \mathbf{S}_{2}\right) \mid \mathbf{S}_{1}\right)=\mathrm{E}\left(\mathbf{U} \mid \mathbf{S}_{1}\right)$ et 
$\mathrm{E}_{\mathrm{S} 1}\left(\mathrm{E}\left(\mathbf{U} \mid \mathbf{S}_{1}, \mathbf{S}_{2}\right) \mid \mathbf{S}_{2}\right)=\mathrm{E}\left(\mathbf{U} \mid \mathbf{S}_{2}\right)$ [Feller, 1971], qui généralisent la relation de l'espérance totale et dans lesquelles la distribution conjointe du couple $\left(\mathbf{S}_{1}, \mathbf{S}_{2}\right)$ intervient. On peut l'étendre à un nombre quelconque de variables avec des vecteurs aléatoires pour $\mathbf{S}_{1}$ et $\mathbf{S}_{2}$ au lieu de variables aléatoires. On a de la sorte une relation générale qui permet d'exprimer une espérance conditionnelle de même nature pour la même réponse aléatoire $\mathbf{U}$, mais avec un nombre de variables de conditionnement plus réduit, après la neutralisation satisfaisante des variables supprimées. L'espérance conditionnelle d'espérances conditionnelles est à l'image de la «sur-sommation» des physiciens quantiques par exemple [Gell-Mann, 1995]. Quelques règles de calcul avec un conditionnement par des variables aléatoires sont rappelées en Annexe 3.

\subsection{2 À propos de l'aléa des stimulations}

La nouvelle présentation des intervalles successifs en $\S 4.2$, avec une variable aléatoire $\mathbf{S}$ pour les expositions, apporte une certaine commodité grâce aux propriétés de l'espérance totale, et celle-ci peut s'étendre aisément au cas multivarié d'un couple $\left(\mathbf{S}_{1}, \mathbf{S}_{2}\right)$ ou d'un K-uple $\left(\mathbf{S}_{1}, \mathbf{S}_{2}, \ldots, \mathbf{S}_{\mathrm{K}}\right)$ de stimulations aléatoires moyennant les propriétés de l'espérance conditionnelle. Pour cela on utilise bien entendu les lois respectives de $\mathbf{S},\left(\mathbf{S}_{1}, \mathbf{S}_{2}\right)$ ou $\left(\mathbf{S}_{1}, \mathbf{S}_{2}, \ldots, \mathbf{S}_{\mathrm{K}}\right)$, et l'on dispose ainsi d'une présentation concise et générale. Par ailleurs, on remarque qu'il s'agit d'un formalisme qui s'applique aussi bien aux variables aléatoires avec des lois de nature probabiliste qu'à des variables prenant plusieurs valeurs avec des distributions de statistique descriptive.

Dans le cas présent, les stimulations suivent des plans d'expérience contrôlés lorsque les expérimentations sont faites en laboratoire et elles pourraient être considérées comme aléatoires quand on échantillonne des personnes exposées au cours d'une enquête in situ comme c'est le cas par exemple pour le niveau de bruit en façade d'habitation. Mais dans tous les cas, quoi qu'il en soit, on dispose du même formalisme performant et concis de l'espérance totale et/ou conditionnelle, avec des calculs selon les distributions statistiques à disposition. À ce titre nous conservons le formalisme probabiliste unificateur doté de ses propriétés bien commodes.

\subsection{L'APPLICATION AUX INTERVALLES SUCCESSIFS}

Ces propriétés se prêtent tout à fait aux résultats des intervalles successifs avec des situations multidimensionnelles ; et, de toute loi conjointe établie en présence de plusieurs variables d'exposition, on peut ainsi en déduire des lois avec moins de variables. Le cas bivarié est celui que nous étudions plus précisément ; à partir d'une réponse conjointe bivariée $\mathrm{E}\left(\mathbf{U} \mid \mathbf{S}_{1}, \mathbf{S}_{2}\right)$, on en déduit les réponses univariées $\mathrm{E}_{\mathrm{S} 2}\left(\mathrm{E}\left(\mathbf{U} \mid \mathbf{S}_{1}, \mathbf{S}_{2}\right) \mid \mathbf{S}_{1}\right)=\mathrm{E}\left(\mathbf{U} \mid \mathbf{S}_{1}\right)$ et $\mathrm{E}_{\mathrm{S} 1}\left(\mathrm{E}\left(\mathbf{U} \mid \mathbf{S}_{1}, \mathbf{S}_{2}\right) \mid \mathbf{S}_{2}\right)=\mathrm{E}\left(\mathbf{U} \mid \mathbf{S}_{2}\right)$, ainsi que les valeurs numériques associées $\mathrm{h}_{12}\left(\mathrm{~s}_{1 \mathrm{i}}, \mathrm{s}_{2 \mathrm{k}}\right)=\mathrm{E}\left(\mathbf{U} \mid \mathbf{S}_{1}=\mathrm{s}_{1 \mathrm{i}}, \mathbf{S}_{2}=\mathrm{s}_{2 \mathrm{k}}\right), \mathrm{h}_{1}\left(\mathrm{~s}_{1 \mathrm{i}}\right)=\mathrm{E}\left(\mathbf{U} \mid \mathbf{S}_{1}=\mathrm{s}_{1 \mathrm{i}}\right)=$ $\mathrm{E}_{\mathrm{S} 2}\left(\mathrm{E}\left(\mathbf{U} \mid \mathbf{S}_{1}=\mathrm{s}_{1 \mathrm{i}}, \mathbf{S}_{2}=\mathrm{s}_{2 \mathrm{k}}\right) \mid \mathbf{S}_{1}=\mathrm{s}_{1 \mathrm{i}}\right)$ et $\mathrm{h}_{2}\left(\mathrm{~s}_{2 \mathrm{k}}\right)=\mathrm{E}\left(\mathbf{U} \mid \mathbf{S}_{2}=\mathrm{s}_{2 \mathrm{k}}\right)=\mathrm{E}_{\mathrm{S} 1}\left(\mathrm{E}\left(\mathbf{U} \mid \mathbf{S}_{1}=\mathrm{s}_{1 \mathrm{i}}\right.\right.$, $\left.\left.\mathbf{S}_{2}=\mathrm{s}_{2 \mathrm{k}}\right) \mid \mathbf{S}_{2}=\mathrm{s}_{2 \mathrm{k}}\right)$.

Ces trois réponses par espérances conditionnelles issues des intervalles successifs dans le cadre multivarié sont sur la même échelle d'intervalle, et en particulier on peut représenter les lois univariées résultantes $\mathrm{E}\left(\mathbf{U} \mid \mathbf{S}_{1}\right)$ et $\mathrm{E}\left(\mathbf{U} \mid \mathbf{S}_{2}\right)$ dans des graphiques avec le même axe des ordonnées. On a ainsi la possibilité de comparer directement les amplitudes respectives $\Delta \mathrm{h}_{1}$ et $\Delta \mathrm{h}_{2}$ qui sont sur une même échelle de rapport, et de noter laquelle des deux variables est la plus agissante dans la réponse conjointe. Cette 
comparabilité des lois univariées après sur-sommation est une conséquence intéressante. En outre lorsque $\mathbf{S}_{1}$ et $\mathbf{S}_{2}$ sont des grandeurs de la même dimension, les graphes de $\mathrm{h}_{1}\left(\mathrm{~s}_{1 \mathrm{i}}\right)$ et $\mathrm{h}_{2}\left(\mathrm{~s}_{2 \mathrm{k}}\right)$ peuvent être figurés dans un même repère de coordonnées, en abscisse comme en ordonnée.

De leur côté les bornes d'intervalle sont données par $t_{j}=E\left(\mathbf{T}_{j} \mid \mathbf{S}_{1}, \mathbf{S}_{2}\right)$, compatibles avec $\mathrm{E}\left(\mathbf{T}_{\mathrm{j}}\right)=\mathrm{E}\left(\mathbf{T}_{\mathrm{j}} \mid \mathbf{S}_{2}\right)=\mathrm{E}_{\mathrm{S} 1}\left(\mathrm{E}\left(\mathbf{T}_{\mathrm{j}} \mid \mathbf{S}_{1}, \mathbf{S}_{2}\right) \mid \mathbf{S}_{2}\right)$ et $\mathrm{E}\left(\mathbf{T}_{\mathrm{j}}\right)=\mathrm{E}\left(\mathbf{T}_{\mathrm{j}} \mid \mathbf{S}_{1}\right)=\mathrm{E}_{\mathrm{S} 2}\left(\mathrm{E}\left(\mathbf{T}_{\mathrm{j}} \mid \mathbf{S}_{1}, \mathbf{S}_{2}\right) \mid \mathbf{S}_{1}\right)$. Ce sont ainsi les mêmes bornes d'intervalle pour les réponses $h_{1}$ et $h_{2}$.

\subsection{ANALOGIES ET DIFFÉRENCES AVEC LE MESURAGE CONJOINT}

Ces résultats apportent une réponse numérique globale en fonction d'une exposition multidimensionnelle et, à ce titre, ils se rapprochent de la démarche du mesurage conjoint comme de celle de l'utilité en fonction d'un panier de plusieurs biens [Beinat, 1995 ; Roberts, 1979 ; Bouyssou, Pirlot, 2002].

i) Le mesurage conjoint dit aussi simultaneous s'est développé peu de temps après le début du mesurage [Luce, Tuckey, 1964 ; Michell, 1990]. Cette extension fait appel à une description multidimensionnelle des situations et elle permet de révéler des représentations numériques en fonction de plusieurs variables ou arguments.

ii) Ce mesurage repose naturellement sur un théorème de représentation, et dans le contexte multidimensionnel la structure des données est essentiellement fondée sur des pré-ordres. Les axiomes sont assez techniques, certains sont du ressort des propriétés topologiques et d'autres sont de ressort structurel de type cancellation [Bouyssou, Pirlot, 2002].

iii) La première application a été celle du mesurage additif conjoint ; les modalités de chaque argument sont représentées par un terme sur une échelle d'intervalle commune, et leur somme $\mathrm{u}_{\mathrm{ikl} 1 . .}=\mathrm{h}\left(\mathrm{s}_{1 \mathrm{i}}, \mathrm{s}_{2 \mathrm{k}}, \mathrm{s}_{31}, \ldots\right)=\mathrm{h}_{1}\left(\mathrm{~s}_{1 \mathrm{i}}\right)+\mathrm{h}_{2}\left(\mathrm{~s}_{2 \mathrm{k}}\right)+\mathrm{h}_{3}\left(\mathrm{~s}_{31}\right)+\ldots$, constitue le mesurage conjoint, avec seulement deux termes la première fois pour Luce et Tuckey. C'est cette même additivité de plusieurs applications univariées à variables séparées que l'on retrouve le plus communément dans la recherche des utilités à plusieurs arguments.

La démarche du mesurage conjoint a ensuite abordé de nombreuses variantes de nature fonctionnelle, comme la decomposability [Roberts, 1979], puis des raffinements dans le cadre de schémas fonctionnels plus précis avec pour chacun d'eux les axiomes spécifiques de plus en plus détaillés [Bouyssou, Pirlot, 2002].

iv) Dans cette veine, avec des échelles de catégories ordonnées et les intervalles successifs, on peut introduire la médiane de la distribution des réponses pour chaque situation $\mathrm{s}_{\{\mathrm{i}\}}$, puis venir se greffer naturellement sur la mise en oeuvre des axiomes structurels du mesurage conjoint moyennant le pré-ordre ainsi construit [Maurin, 1986].

v) D'une manière différente, les intervalles successifs dans le contexte multivarié permettent ici une solution conjointe alternative qui ne met en oeuvre que le seul respect des axiomes d'Adams et Messick, et qui est de facto libérée du poids des axiomes spécifiques à des schémas fonctionnels précis. Cela ouvre ainsi l'accès à un ensemble beaucoup plus vaste de relations sans schéma analytique particulier de référence, tout en bénéficiant des propriétés commodes des espérances conditionnelles multiples (cf. Figure 6). 


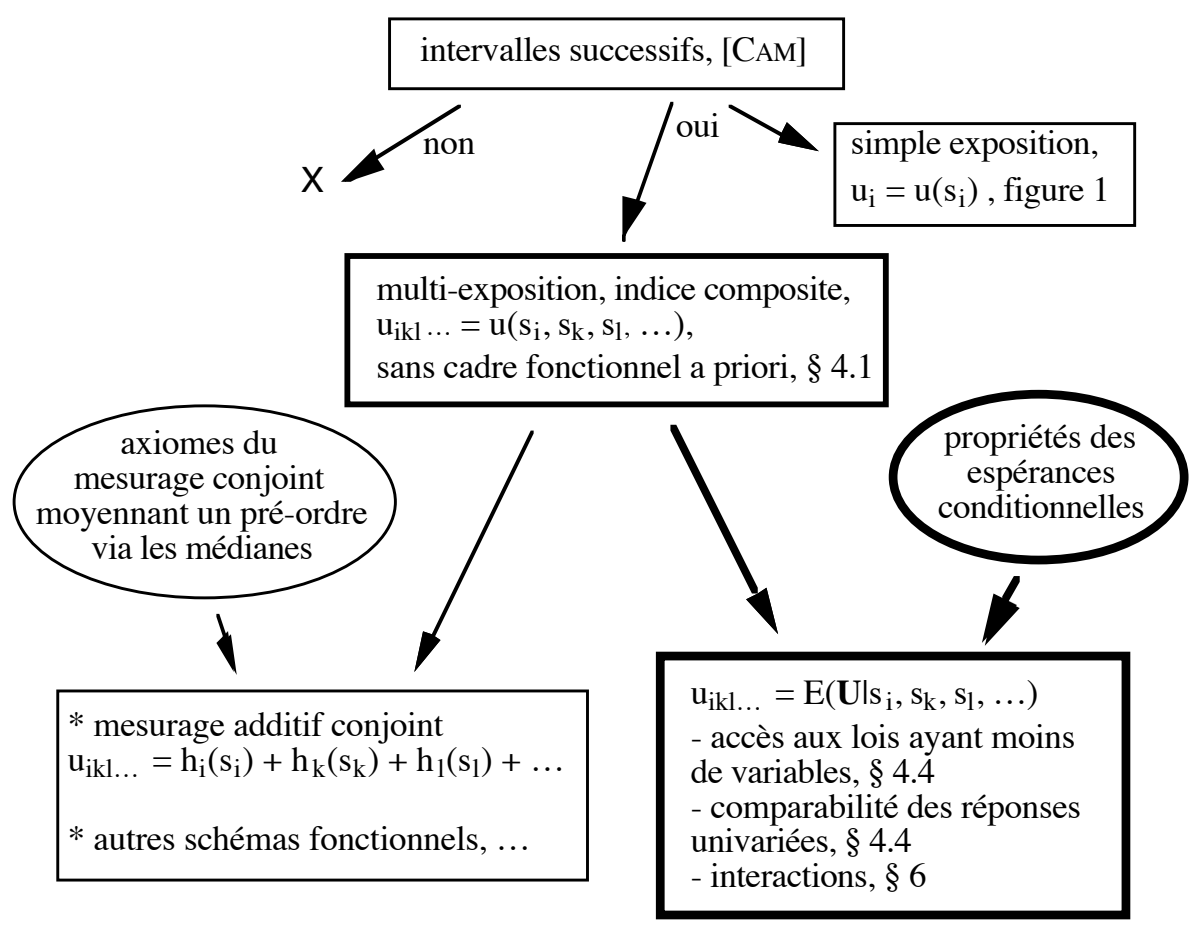

FIGURE 6. Analogies et différences entre le mesurage conjoint et les intervalles successifs dans un contexte multivarié

\section{UN PREMIER EXEMPLE DE BI-EXPOSITION}

\section{a) Les données}

Il s'agit de réponses de gêne pour des résidents dont les habitations sont exposées à des bruits provenant de deux sources différentes. La gêne globale $\mathbf{U}$ est recueillie à l'aide d'une échelle à 4 catégories, et les traitements sonométriques faits pendant les enregistrements permettent de calculer les niveaux de bruit $\mathbf{S}_{1}$ et $\mathbf{S}_{2}$ de chacune des deux sources, avec des niveaux respectifs $\mathrm{s}_{1 \mathrm{i}}, \mathrm{i}=1 \ldots 5$, pour $\mathbf{S}_{1}$, et $\mathrm{s}_{2 \mathrm{k}}, \mathrm{k}=1 \ldots 5$, (niveaux rangés dans des plages de 3 décibels d'étendue). Les mesures étant faites in situ le plan d'expérience des variables $\mathbf{S}_{1}$ et $\mathbf{S}_{2}$ résulte des mesures effectuées au cours de l'étude, et l'on a 19 situations exploitables pour un effectif total de 622 personnes, (les biexpositions fortes pour une source et faible pour l'autre ne se sont pas présentées dans l'enquête et ne figurent donc pas dans le plan) [Maurin, 2001].

\begin{tabular}{|c|c|c|c|c|c|c|}
\hline $\mathbf{S}_{2}$ & $\mathrm{~s}_{21}$ & $\mathrm{~s}_{22}$ & $\mathrm{~s}_{23}$ & $\mathrm{~s}_{24}$ & $\mathrm{~s}_{25}$ & $\mathrm{n}_{+\mathrm{k}}$ \\
\hline $\mathbf{S}_{1}: \mathrm{s}_{11}$ & & 18 & 23 & & & 41 \\
\hline $\mathrm{s}_{12}$ & 16 & 37 & 38 & 17 & & 108 \\
\hline$s_{13}$ & 18 & 44 & 48 & 46 & 18 & 174 \\
\hline $\mathrm{s}_{14}$ & 30 & 39 & 33 & 48 & 32 & 182 \\
\hline $\mathrm{s}_{15}$ & & & 20 & 42 & 55 & 117 \\
\hline $\mathrm{n}_{\mathrm{i}+}$ & 64 & 138 & 162 & 153 & 105 & 622 \\
\hline
\end{tabular}

TABLEAU 4. Le plan des variables d'exposition et les effectifs de répondants $n_{\mathrm{ik}}$ 


\section{b) Le mesurage}

On établit le tableau de contingence associé à 19 lignes pour les expositions et 4 colonnes pour les catégories de réponse sur lequel on applique les axiomes $\left[\mathrm{C}_{\mathrm{AM}}\right]$. Ceux-ci étant raisonnablement vérifiés avec la fonction de répartition de la loi logistique, l'algorithme conduit aux 19 valeurs $\mathrm{u}_{\mathrm{ik}}=\mathrm{E}\left(\mathbf{U} \mid \mathbf{S}_{1}=\mathrm{s}_{1 \mathrm{i}}, \mathbf{S}_{2}=\mathrm{s}_{2 \mathrm{k}}\right)$ qui sont les mesurages des $\left(s_{1 i}, s_{2 k}\right)$, aux bornes $t_{j}$ et aux barycentres $c_{j}$, (Tableau 5 pour les seuls $\left.\mathrm{u}_{\mathrm{ik}}\right)$.

\begin{tabular}{l|ccccc|}
\multicolumn{1}{c}{} & $\mathrm{s}_{21}$ & $\mathrm{~s}_{22}$ & $\mathrm{~s}_{23}$ & $\mathrm{~s}_{24}$ & $\mathrm{~s}_{25}$ \\
\cline { 2 - 6 } $\mathrm{s}_{11}$ & & 6,37 & 9,69 & & \\
$\mathrm{~s}_{12}$ & 1,00 & 11,44 & 16,96 & 13,63 & \\
$\mathrm{~s}_{13}$ & 6,97 & 8,34 & 17,44 & 18,02 & 13,54 \\
$\mathrm{~s}_{14}$ & 5,37 & 8,87 & 14,9 & 20,17 & 22,14 \\
$\mathrm{~s}_{15}$ & & & 13,85 & 16,81 & 19,00 \\
\cline { 3 - 6 } & & & & &
\end{tabular}

TABLEAU 5. Les mesurages numériques $\mathrm{u}_{\mathrm{ik}}$ de la bi-exposition

c) Les lois des expositions univariées sur-sommées

On en déduit les réponses aux expositions univariées à partir des espérances conditionnelles, $\S 4.4$

$\begin{array}{lrcccc}\mathbf{S}_{1}: & \mathrm{s}_{11} & \mathrm{~s}_{12} & \mathrm{~s}_{13} & \mathrm{~s}_{14} & \mathrm{~s}_{15} \\ \mathrm{u}_{1 \mathrm{i}}: & 8,24 & 12,18 & 13,80 & 14,70 & 17,33 \\ \mathbf{S}_{2}: & \mathrm{s}_{21} & \mathrm{~s}_{22} & \mathrm{~s}_{23} & \mathrm{~s}_{24} & \mathrm{~s}_{25} \\ \mathrm{u}_{2 \mathrm{k}}: & 4,73 & 9,06 & 15,27 & 17,87 & 19,02\end{array}$

sur une échelle d'intervalle commune avec la même espérance $\mathrm{E}(\mathbf{U})=14,08$.

On peut les représenter dans le même graphe puisque l'ordonnée est la même par construction, et que l'abscisse correspond à l'unité commune des deux expositions (des niveaux de bruit exprimés en décibels) (cf. Figure 7).

Les deux lois de réponse sont croissantes, pour les faibles valeurs de bruit la réponse en fonction de la source $\mathbf{S}_{1}$ est supérieure à la réponse en fonction de $\mathbf{S}_{2}$, et elles se rejoignent un peu au-dessus de 60 décibels. La première réponse (en $\mathbf{S}_{1}$ ) croît assez régulièrement sans trop de changement de pente, alors que la réponse en $\mathbf{S}_{2}$ présente une forte accélération dans la première partie de sa croissance avant de ralentir. L'étendue $\Delta \mathrm{h}_{2}$ de la réponse en $\mathbf{S}_{2}$ est égale à 14,29 et l'étendue $\Delta \mathrm{h}_{1}$ en fonction de $\mathbf{S}_{1}$ est égale à 9,09 , soit $65 \%$ de la précédente.

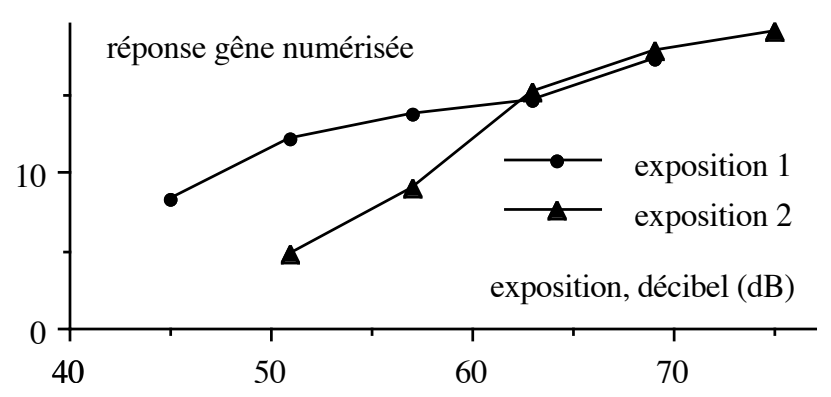

FIGURE 7. Les correspondances univariées après sur-sommation de la réponse conjointe 


\section{LA RÉPONSE CONJOINTE ET L'INTERACTION ENTRE VARIABLES}

Il s'avère en outre que la formulation particulière $\mathrm{E}\left(\mathbf{U} \mid \mathbf{S}_{1}, \mathbf{S}_{2}\right)$ de la réponse conjointe permet d'aller au-delà, et d'examiner commodément plusieurs de ses propriétés. En effet $\mathrm{E}\left(\mathbf{U} \mid \mathbf{S}_{1}, \mathbf{S}_{2}\right)$ est une variable aléatoire transformée $\mathrm{g}\left(\mathbf{S}_{1}, \mathbf{S}_{2}\right)$ de deux variables aléatoires, une transformée dans laquelle intervient la distribution conjointe de probabilité du couple $\left(\mathbf{S}_{1}, \mathbf{S}_{2}\right)$. Cela permet notamment d'en déduire son espérance et sa variance, (Annexe 4).

\subsection{L'INTERACTION ENTRE VARIABLES DANS E( $\left(\mathbf{U} \mid \mathbf{S}_{1}, \mathbf{S}_{2}\right)$}

En second, on remarque que les propriétés des espérances conditionnelles permettent d'introduire et d'étudier l'interaction entre les variables $\mathbf{S}_{1}$ et $\mathbf{S}_{2}$ dans la réponse conjointe.

\subsubsection{Une définition classique}

Les premiers développements du mesurage conjoint et les développements ordinaires de l'utilité privilégient, a-t-on vu, une réponse conjointe additive de termes univariés [Beinat, 1995; Roberts, 1979; Roy, Bouyssou, 1993]. C'est une somme d'effets unifactoriels comme celle-ci qui se rencontre également dans l'analyse de la variance des tableaux de contingence multivariés sans interaction, tandis que dans les «plans avec interaction» on fait figurer un terme dépendant de plusieurs variables en complément à la somme précédente [Chapouille, 1973].

Ceci invite à décomposer la réponse conjointe sous la forme

$$
\mathrm{E}\left(\mathbf{U} \mid \mathbf{S}_{1}, \mathbf{S}_{2}\right)=\mathrm{E}\left(\mathbf{U} \mid \mathbf{S}_{1}\right)+\mathrm{E}\left(\mathbf{U} \mid \mathbf{S}_{2}\right)-\mathrm{E}(\mathbf{U})+\mathrm{c}\left(\mathbf{S}_{1}, \mathbf{S}_{2}\right),
$$

ce qui revient à définir le terme d'interaction

$$
\mathrm{c}\left(\mathbf{S}_{1}, \mathbf{S}_{2}\right)=\mathrm{E}\left(\mathbf{U} \mid \mathbf{S}_{1}, \mathbf{S}_{2}\right)-\mathrm{E}\left(\mathbf{U} \mid \mathbf{S}_{1}\right)-\mathrm{E}\left(\mathbf{U} \mid \mathbf{S}_{2}\right)+\mathrm{E}(\mathbf{U}) \quad[\mathrm{cg}]
$$

Ce terme présente une interprétation phénoménologique immédiate. En effet une interaction positive est le signe que la réponse conjointe est supérieure à la simple somme des effets univariés, c'est-à-dire qu'il y a un effet d'entraînement, d'amplification ou de renforcement mutuel entre les variables, ou encore que la réponse est « sur-additive » par rapport au cumul des seuls effets individuels pris isolément. À l'inverse une interaction négative est le signe d'un effet de saturation ou d'une forme de retenue ou d'inhibition dans la réponse globale. Ce terme complémentaire présente une forte lisibilité, et c'est sans doute pour cela qu'il est introduit sans argumentation particulière [Job, Hatfield, 2001]. Ce terme est aussi par construction la quantité à laquelle sont dus les défauts de parallélisme entre les différentes coupes partielles de la réponse conjointe quand l'un des deux arguments est constant, $\S 4.1$.

\subsubsection{Les premières propriétés de l'interaction}

LEMME 1. Le terme d'interaction $\mathrm{c}\left(\mathbf{S}_{1}, \mathbf{S}_{2}\right)$ et ses espérances conditionnelles sont centrées pour la loi du couple $\left(\mathbf{S}_{1}, \mathbf{S}_{2}\right)$.

On remarque en effet que tous les termes de $\mathrm{c}\left(\mathbf{S}_{1}, \mathbf{S}_{2}\right)$ dans [cg] ont la même espérance $\mathrm{E}(\mathbf{U})$ pour la loi de $\left(\mathbf{S}_{1}, \mathbf{S}_{2}\right)$, par conséquent l'interaction c est centrée, c'est-àdire $\mathrm{E}\left(\mathrm{c}\left(\mathbf{S}_{1}, \mathbf{S}_{2}\right)\right)=0$. 
Naturellement on a $\mathrm{E}\left(\mathrm{c}\left(\mathbf{S}_{1}, \mathbf{S}_{2}\right)\right)=\mathrm{E}_{\mathrm{S} 2}\left(\mathrm{E}_{\mathrm{S} 1}\left(\mathrm{c}\left(\mathbf{S}_{1}, \mathbf{S}_{2}\right)\right) \mid \mathbf{S}_{2}\right)=\mathrm{E}_{\mathrm{S} 1}\left(\mathrm{E}_{\mathrm{S} 2}\left(\mathrm{c}\left(\mathbf{S}_{1}, \mathbf{S}_{2}\right)\right) \mid \mathbf{S}_{1}\right)$, et les espérances conditionnelles $\mathrm{c}_{1}\left(\mathbf{S}_{1}\right)=\mathrm{E}_{\mathrm{S} 2}\left(\mathrm{c}\left(\mathbf{S}_{1}, \mathbf{S}_{2}\right) \mid \mathbf{S}_{1}\right)$ et $\mathrm{c}_{2}\left(\mathbf{S}_{2}\right)=\mathrm{E}_{\mathrm{S} 1}\left(\mathrm{c}\left(\mathbf{S}_{1}, \mathbf{S}_{2}\right) \mid \mathbf{S}_{2}\right)$ de $\mathrm{c}\left(\mathbf{S}_{1}, \mathbf{S}_{2}\right)$ sont elles-mêmes centrées puisque $\mathrm{E}\left(\mathrm{c}\left(\mathbf{S}_{1}, \mathbf{S}_{2}\right)\right)=\mathrm{E}_{\mathrm{S} 1}\left(\mathrm{c}_{1}\left(\mathbf{S}_{1}\right)\right)=\mathrm{E}_{\mathrm{S} 2}\left(\mathrm{c}_{2}\left(\mathbf{S}_{2}\right)\right)$.

La propriété ci-dessus est propre aux espérances conditionnelles en général, si l'on revient aux intervalles successifs proprement dits on en déduit un résultat de mesurage :

LEMME 2. Dans le cadre des solutions d'une application des intervalles successifs multivariés, le terme d'interaction prend ses valeurs sur une échelle de rapport.

En effet à la suite des intervalles successifs la réponse conjointe $\mathrm{E}\left(\mathbf{U} \mid \mathbf{S}_{1}, \mathbf{S}_{2}\right)$ et les autres espérances qui en résultent par les prises d'espérances conditionnelles prennent leur valeur sur une échelle d'intervalle commune. Il en est de même des sommes partielles $\mathrm{E}\left(\mathbf{U} \mid \mathbf{S}_{1}, \mathbf{S}_{2}\right)+\mathrm{E}(\mathbf{U})$ et $\mathrm{E}\left(\mathbf{U} \mid \mathbf{S}_{1}\right)+\mathrm{E}\left(\mathbf{U} \mid \mathbf{S}_{2}\right)$, et le terme constant disparaît dans la différence de ces deux quantités. L'échelle de rapport qui en résulte a la même constante multiplicative que l'échelle d'intervalle des divers $\mathrm{E}(\mathbf{U} \mathrm{l} . .$.$) .$

\subsubsection{L'ordre de grandeur numérique de l'interaction}

Pour les valeurs de conditionnement connues $s_{1 i}$ et $s_{2 k}$ du plan expérimental des expositions, on a naturellement les valeurs numériques des réponses en ces points, bivariée avec $\mathrm{E}\left(\mathbf{U} \mid \mathbf{S}_{1}=\mathrm{s}_{1 \mathrm{i}}, \mathbf{S}_{2}=\mathrm{s}_{2 \mathrm{k}}\right)$ et univariées avec $\mathrm{E}\left(\mathbf{U} \mid \mathbf{S}_{1}=\mathrm{s}_{1 \mathrm{i}}\right), \mathrm{E}\left(\mathbf{U} \mid \mathbf{S}_{2}=\mathrm{s}_{2 \mathrm{k}}\right)$. On en déduit aussitôt les valeurs numériques de l'interaction $\mathrm{c}\left(\mathrm{s}_{1 \mathrm{i}}, \mathrm{s}_{2 \mathrm{k}}\right)=$ $\mathrm{E}\left(\mathbf{U} \mid \mathbf{S}_{1}=\mathrm{s}_{1 \mathrm{i}}, \mathbf{S}_{2}=\mathrm{s}_{2 \mathrm{k}}\right)-\mathrm{E}\left(\mathbf{U} \mid \mathbf{S}_{1}=\mathrm{s}_{1 \mathrm{i}}\right)-\mathrm{E}\left(\mathbf{U} \mid \mathbf{S}_{2}=\mathrm{s}_{2 \mathrm{k}}\right)+\mathrm{E}(\mathbf{U})$ pour les mêmes modalités de $\mathbf{S}_{1}$ et $\mathbf{S}_{2}$.

Sur le plan qualitatif, on a noté que la non nullité et le signe du terme d'interaction ont une interprétation immédiate, $\S 6.1 .1$. De leur côté, les valeurs numériques permettent d'étalonner de manière non moins immédiate l'importance relative de ce terme par rapport à la réponse. En effet, les diverses amplitudes numériques $\Delta \mathrm{h}_{\mathrm{r}} \mathrm{r}=1,2$ des lois univariées qui sont obtenues par les espérances conditionnelles de la solution globale sont comparables entre elles, $\S 4.4$, et le Lemme 2 permet ici de comparer les valeurs du terme d'interaction à ces amplitudes ou à toute autre valeur de référence comme $\left(\Delta \mathrm{h}_{1}+\Delta \mathrm{h}_{2}\right) / 2$ par exemple.

\subsection{LA VARIANCE DE L'INTERACTION}

On a également la possibilité d'expertiser l'ordre de grandeur de l'interaction avec une autre décomposition de la variance de la réponse que la décomposition classique de l'Annexe 4. On pose ici les notations allégées $\mathbf{U}_{12}=\mathrm{E}\left(\mathbf{U} \mid \mathbf{S}_{1}, \mathbf{S}_{2}\right), \mathbf{U}_{1}=\mathrm{E}\left(\mathbf{U} \mid \mathbf{S}_{1}\right), \mathbf{U}_{2}=$ $\mathrm{E}\left(\mathbf{U} \mid \mathbf{S}_{2}\right)$ et $\mathbf{c}=\mathbf{U}_{12}-\mathbf{U}_{1}-\mathbf{U}_{2}+\mathrm{E}(\mathbf{U})$.

THÉORÈME 1. On a la décomposition algébrique $\operatorname{var}\left(\mathbf{U}_{12}\right)=\operatorname{var}\left(\mathbf{U}_{1}-\mathbf{U}_{2}\right)+\operatorname{var}(\mathbf{c})$.

En effet, la définition $\mathbf{U}_{12}=\mathbf{U}_{1}+\mathbf{U}_{2}-\mathrm{E}(\mathbf{U})+\mathbf{c}$ donne

$$
\operatorname{var}\left(\mathbf{U}_{12}\right)=\operatorname{var}\left(\mathbf{U}_{1}\right)+\operatorname{var}\left(\mathbf{U}_{2}\right)+\operatorname{var}(\mathbf{c})+2 \operatorname{cov}\left(\mathbf{U}_{1}, \mathbf{U}_{2}\right)+2 \operatorname{cov}\left(\mathbf{U}_{1}, \mathbf{c}\right)+2 \operatorname{cov}\left(\mathbf{U}_{2}, \mathbf{c}\right) .
$$

Quand on remplace c par sa définition dans les covariances, on obtient

a) pour $\mathbf{U}_{1}: \operatorname{cov}\left(\mathbf{U}_{1}, \mathbf{c}\right)=\operatorname{cov}\left(\mathbf{U}_{1}, \mathbf{U}_{12}-\mathbf{U}_{1}-\mathbf{U}_{2}\right)=-\operatorname{cov}\left(\mathbf{U}_{1}, \mathbf{U}_{2}\right)+\operatorname{cov}\left(\mathbf{U}_{1}, \mathbf{U}_{12}-\mathbf{U}_{1}\right)$;

on a $\operatorname{cov}\left(\mathbf{U}_{1}, \mathbf{U}_{12}-\mathbf{U}_{1}\right)=\mathrm{E}_{\mathrm{S} 1}\left[\mathrm{E}_{\mathrm{S} 2}\left\{\left(\mathbf{U}_{1}-\mathrm{E}(\mathbf{U})\right) .\left(\mathbf{U}_{12}-\mathbf{U}_{1}\right) \mid \mathbf{S}_{1}\right\}\right]=\mathrm{E}_{\mathrm{S} 1}\left[\left(\mathbf{U}_{1}-\mathrm{E}(\mathbf{U})\right)\right.$. $\left.\mathrm{E}_{\mathrm{S} 2}\left\{\left(\mathbf{U}_{12}-\mathbf{U}_{1}\right) \mid \mathbf{S}_{1}\right\}\right]$, l'espérance $\mathrm{E}_{\mathrm{S} 2}\left\{\left(\mathbf{U}_{12}-\mathbf{U}_{1}\right) \mid \mathbf{S}_{1}\right\}$ est nulle puisque $\mathbf{U}_{1}=\mathrm{E}_{\mathrm{S} 2}\left(\mathbf{U}_{12} \mid \mathbf{S}_{1}\right)$

et l'on en déduit $\operatorname{cov}\left(\mathbf{U}_{1}, \mathbf{c}\right)=-\operatorname{cov}\left(\mathbf{U}_{1}, \mathbf{U}_{2}\right)$. 
b) On a l'équivalent pour $\mathbf{U}_{2}$,

c) il en résulte que $\operatorname{cov}\left(\mathbf{U}_{1}, \mathbf{c}\right)+\operatorname{cov}\left(\mathbf{U}_{2}, \mathbf{c}\right)=-2 \operatorname{cov}\left(\mathbf{U}_{1}, \mathbf{U}_{2}\right)$, et par conséquent

$$
\operatorname{var}\left(\mathbf{U}_{12}\right)=\operatorname{var}\left(\mathbf{U}_{1}\right)+\operatorname{var}\left(\mathbf{U}_{2}\right)-2 \operatorname{cov}\left(\mathbf{U}_{1}, \mathbf{U}_{2}\right)+\operatorname{var}(\mathbf{c}) .
$$

Cette décomposition permet ainsi de connaître la part de la variance de l'interaction dans la variance de la réponse globale.

\subsection{UNE DÉCOMPOSITION FORMELLE POUR E $\left(\mathbf{U} I \mathbf{S}_{1}, \mathbf{S}_{2}\right)$}

Le terme d'interaction du $\S 6.1 .1$ est posé a priori grâce à sa commodité d'interprétation, mais on peut aussi le retrouver d'une manière directe, indépendamment de ce volet intuitif.

\subsubsection{Un terme de référence sous l'indépendance}

THÉORÈME 2. Lorsque les variables $\mathbf{S}_{1}$ et $\mathbf{S}_{2}$ sont indépendantes, l'espérance conditionnelle $\mathrm{E}\left(\mathbf{U} \mid \mathbf{S}_{1}, \mathbf{S}_{2}\right)$ est la somme de deux termes $\mathrm{a}_{1}\left(\mathbf{S}_{1}\right)$ et $\mathrm{a}_{2}\left(\mathbf{S}_{2}\right)$ à variables séparées et d'une constante $\mathrm{C}$ si et seulement si on a la relation $\mathrm{E}\left(\mathbf{U I} \mathbf{S}_{1}, \mathbf{S}_{2}\right)=\mathrm{E}\left(\mathbf{U} \mid \mathbf{S}_{1}\right)+$ $\mathrm{E}\left(\mathbf{U} \mid \mathbf{S}_{2}\right)-\mathrm{E}(\mathbf{U})$.

La condition suffisante est évidente puisque les $\mathrm{E}\left(\mathbf{U} \mid \mathbf{S}_{\mathrm{r}}\right)$ sont des transformations respectives de $\mathbf{S}_{\mathrm{r}}, \mathrm{r}=1,2$, indépendantes ou non.

Pour la condition nécessaire, on suppose que $\mathrm{E}\left(\mathbf{U} \mid \mathbf{S}_{1}, \mathbf{S}_{2}\right)$ est de la forme $\mathrm{a}_{1}\left(\mathbf{S}_{1}\right)+\mathrm{a}_{2}\left(\mathbf{S}_{2}\right)+\mathrm{C}$. Quand on en prend l'espérance conditionnelle, par exemple $\mathrm{E}_{\mathrm{S} 2}\left(\mathrm{E}\left(\mathbf{U} \mid \mathbf{S}_{1}, \mathbf{S}_{2}\right) \mid \mathbf{S}_{1}\right)=\mathrm{E}\left(\mathbf{U} \mid \mathbf{S}_{1}\right)$, on a le développement

$$
\mathrm{E}_{\mathrm{S} 2}\left(\mathrm{a}_{1}\left(\mathbf{S}_{1}\right) \mid \mathbf{S}_{1}\right)+\mathrm{E}_{\mathrm{S} 2}\left(\mathrm{a}_{2}\left(\mathbf{S}_{2}\right) \mid \mathbf{S}_{1}\right)+\mathrm{C} .
$$

Le premier terme du membre de droite est l'espérance en $\mathbf{S}_{2}$ d'une quantité qui ne dépend pas de $\mathbf{S}_{2}$, c'est donc cette quantité a $\left(\mathbf{S}_{1}\right)$, le second fait intervenir la loi conjointe de $\left(\mathbf{S}_{1}, \mathbf{S}_{2}\right)$ et dans le cas de l'indépendance il se simplifie et devient égal à l'espérance $\mathrm{E}_{\mathrm{S} 2}\left(\mathrm{a}_{2}\left(\mathbf{S}_{2}\right)\right)$.

On a donc $\mathrm{E}\left(\mathbf{U} \mid \mathbf{S}_{1}\right)=\mathrm{a}_{1}\left(\mathbf{S}_{1}\right)+\mathrm{ES}_{\mathrm{S} 2}\left(\mathrm{a}_{2}\left(\mathbf{S}_{2}\right)\right)+\mathrm{C}$,

et de manière analogue, on a $\mathrm{E}_{\mathrm{S} 1}\left(\mathrm{E}\left(\mathbf{U} \mid \mathbf{S}_{1}, \mathbf{S}_{2}\right) \mid \mathbf{S}_{2}\right)=\mathrm{E}\left(\mathbf{U} \mid \mathbf{S}_{2}\right)=\mathrm{a}_{2}\left(\mathbf{S}_{2}\right)+\mathrm{E}_{\mathrm{S} 1}\left(\mathrm{a}_{1}\left(\mathbf{S}_{1}\right)\right)+\mathrm{C}$ sous l'indépendance, d'où il résulte que

$\mathrm{E}\left(\mathbf{U} \mid \mathbf{S}_{1}\right)+\mathrm{E}\left(\mathbf{U} \mid \mathbf{S}_{2}\right)=\mathrm{a}_{1}\left(\mathbf{S}_{1}\right)+\mathrm{a}_{2}\left(\mathbf{S}_{2}\right)+\mathrm{E}_{\mathrm{S} 1}\left(\mathrm{a}_{1}\left(\mathbf{S}_{1}\right)\right)+\mathrm{E}_{\mathrm{S} 2}\left(\mathrm{a}_{2}\left(\mathbf{S}_{2}\right)\right)+2 \mathrm{C}$, c'est-à-dire $\mathrm{E}\left(\mathbf{U} \mid \mathbf{S}_{1}\right)+\mathrm{E}\left(\mathbf{U} \mid \mathbf{S}_{2}\right)=\mathrm{E}\left(\mathbf{U} \mid \mathbf{S}_{1}, \mathbf{S}_{2}\right)+\mathrm{E}_{\mathrm{S} 1}\left(\mathrm{a}_{1}\left(\mathbf{S}_{1}\right)\right)+\mathrm{E}_{\mathrm{S} 2}\left(\mathrm{a}_{2}\left(\mathbf{S}_{2}\right)\right)+\mathrm{C}$.

Cela conduit donc à l'identification terme à terme $\mathrm{a}_{1}\left(\mathbf{S}_{1}\right)=\mathrm{E}\left(\mathbf{U} \mid \mathbf{S}_{1}\right), \mathrm{a}_{2}\left(\mathbf{S}_{2}\right)=$ $\mathrm{E}\left(\mathbf{U} \mid \mathbf{S}_{2}\right)$ et $\mathrm{C}=-\mathrm{E}_{\mathrm{S} 1}\left(\mathrm{a}_{1}\left(\mathbf{S}_{1}\right)\right)-\mathrm{E}_{\mathrm{S} 2}\left(\mathrm{a}_{2}\left(\mathbf{S}_{2}\right)\right)-\mathrm{C}$, puis à $\mathrm{C}=-\mathrm{E}(\mathbf{U})$.

Par conséquent dans le cas de l'indépendance de la distribution des variables d'exposition $\left(\mathbf{S}_{1}, \mathbf{S}_{2}\right)$, le théorème 2 explicite un terme de référence additif à variables séparées pour le développement de la réponse conjointe $d_{\text {ref }}=\mathrm{E}\left(\mathbf{U} \mid \mathbf{S}_{1}\right)+\mathrm{E}\left(\mathbf{U} \mid \mathbf{S}_{2}\right)-\mathrm{E}(\mathbf{U})$. Cela revient à poser la définition

$$
\mathrm{c}_{\text {indep }}\left(\mathbf{S}_{1}, \mathbf{S}_{2}\right)=\mathrm{E}\left(\mathbf{U} \mid \mathbf{S}_{1}, \mathbf{S}_{2}\right)-\mathrm{d}_{\text {ref }} \quad\left[\mathrm{c}_{\text {indep }}\right]
$$

qui valide formellement la définition classique [cg]. Cependant, avec l'indépendance, l'expression du terme d'interaction résulte d'une condition nécessaire et suffisante au lieu d'être simplement suggérée par la lisibilité de l'interprétation. 


\subsubsection{Des propriétés complémentaires}

LEMME 3. Lorsque les variables $\mathbf{S}_{1}$ et $\mathbf{S}_{2}$ sont indépendantes les espérances conditionnelles du terme d'interaction $\mathrm{c}_{\mathrm{indep}}\left(\mathbf{S}_{1}, \mathbf{S}_{2}\right)$ sont identiquement nulles.

On a ici cindep1 $\left(\mathbf{S}_{1}\right)=\mathrm{E}_{\mathrm{S} 2}\left(\mathrm{c}_{\text {indep }}\left(\mathbf{S}_{1}, \mathbf{S}_{2}\right) \mid \mathbf{S}_{1}\right)=\mathrm{E}_{\mathrm{S} 2}\left(\mathrm{E}\left(\mathbf{U} \mid \mathbf{S}_{1}, \mathbf{S}_{2}\right) \mid \mathbf{S}_{1}\right)-\mathrm{E}_{\mathrm{S} 2}\left(\mathrm{E}\left(\mathbf{U} \mid \mathbf{S}_{1}\right) \mid \mathbf{S}_{1}\right)-$ $\mathrm{E}_{\mathrm{S} 2}\left(\mathrm{E}\left(\mathbf{U} \mid \mathbf{S}_{2}\right) \mid \mathbf{S}_{1}\right)+\mathrm{E}_{\mathrm{S} 2}\left(\mathrm{E}(\mathbf{U}) \mid \mathbf{S}_{1}\right)$.

Les calculs se font ici en revenant aux diverses quantités $\mathrm{E}(\mathbf{U I} . .$.$) . Le premier$ terme de $\mathrm{c}_{\text {indep1 }}\left(\mathbf{S}_{1}\right)$ est égal à $\mathrm{E}\left(\mathbf{U} \mid \mathbf{S}_{1}\right)$, le second est l'espérance en $\mathbf{S}_{2}$ de $\mathrm{E}\left(\mathbf{U} \mid \mathbf{S}_{1}\right)$ qui ne dépend pas de $\mathbf{S}_{2}$, c'est donc à nouveau $\mathrm{E}\left(\mathbf{U} \mid \mathbf{S}_{1}\right)$, et le dernier est la constante $\mathrm{E}(\mathbf{U})$. Quant au troisième, son développement est simple grâce à l'indépendance, en effet $\mathrm{E}_{\mathrm{S} 2}\left(\mathrm{E}\left(\mathbf{U} \mid \mathbf{S}_{2}\right) \mid \mathbf{S}_{1}\right)=\mathrm{E}_{\mathrm{S} 2}\left(\mathrm{E}\left(\mathbf{U} \mid \mathbf{S}_{2}\right)=\mathrm{E}(\mathbf{U})\right.$. On a donc bien $\mathrm{c}_{\text {indep } 1}\left(\mathbf{S}_{1}\right)=0$.

Naturellement on a de la même façon

$$
\mathrm{c}_{\text {indep } 2}\left(\mathbf{S}_{2}\right)=\mathrm{E}_{\mathrm{S} 1}\left(\mathrm{c}_{\text {indep }}\left(\mathbf{S}_{1}, \mathbf{S}_{2}\right) \mid \mathbf{S}_{2}\right)=0 \text {. }
$$

Les deux interactions $\mathrm{c}\left(\mathbf{S}_{1}, \mathbf{S}_{2}\right)$ et $\mathrm{c}_{\text {indep }}\left(\mathbf{S}_{1}, \mathbf{S}_{2}\right)$ sont donc centrées, et leurs espérances conditionnelles également. En outre l'indépendance de la distribution de $\left(\mathbf{S}_{1}, \mathbf{S}_{2}\right)$ entraîne que les espérances conditionnelles sont identiquement nulles.

\subsubsection{Le cas de la non-indépendance}

Dans la pratique on ne peut cependant pas négliger une dépendance dans la loi conjointe du plan $\left(\mathbf{S}_{1}, \mathbf{S}_{2}\right)$. Nous reprenons ici un développement de la forme $\mathrm{E}\left(\mathbf{U} \mid \mathbf{S}_{1}, \mathbf{S}_{2}\right)=\mathrm{a}_{1}\left(\mathbf{S}_{1}\right)+$ $\mathrm{a}_{2}\left(\mathbf{S}_{2}\right)+\mathrm{C}$ pour le cas général.

THÉORÈME 3. Pour qu'un développement $\mathrm{a}_{1}\left(\mathbf{S}_{1}\right)+\mathrm{a}_{2}\left(\mathbf{S}_{2}\right)+\mathrm{C}$ additif à variables séparées de $\mathrm{E}\left(\mathbf{U} \mid \mathbf{S}_{1}, \mathbf{S}_{2}\right)$ ait lieu dans le cas général de la dépendance, il est nécessaire que $\mathrm{C}$ soit nul et que les deux applications $\mathrm{a}_{1}\left(\mathbf{S}_{1}\right)$ et $\mathrm{a}_{2}\left(\mathbf{S}_{2}\right)$ vérifient le système :

$$
\begin{aligned}
& \mathrm{a}_{1}\left(\mathbf{S}_{1}\right)=\mathrm{E}\left(\mathbf{U} \mid \mathbf{S}_{1}\right)-\mathrm{E}_{\mathrm{S} 2}\left(\mathrm{a}_{2}\left(\mathbf{S}_{2}\right) \mid \mathbf{S}_{1}\right) \\
& \mathrm{a}_{2}\left(\mathbf{S}_{2}\right)=\mathrm{E}\left(\mathbf{U} \mid \mathbf{S}_{2}\right)-\mathrm{E}_{\mathrm{S} 1}\left(\mathrm{a}_{1}\left(\mathbf{S}_{1}\right) \mid \mathbf{S}_{2}\right) .
\end{aligned}
$$

En effet avec ce développement, on a comme dans le Théorème 2

$$
\begin{aligned}
& \mathrm{E}_{\mathrm{S} 2}\left(\mathrm{E}\left(\mathbf{U} \mid \mathbf{S}_{1}, \mathbf{S}_{2}\right) \mid \mathbf{S}_{1}\right)=\mathrm{E}\left(\mathbf{U} \mid \mathbf{S}_{1}\right)=\mathrm{a}_{1}\left(\mathbf{S}_{1}\right)+\mathrm{E}_{\mathrm{S} 2}\left(\mathrm{a}_{2}\left(\mathbf{S}_{2}\right) \mid \mathbf{S}_{1}\right)+\mathrm{C} \\
& \mathrm{E}_{\mathrm{S} 1}\left(\mathrm{E}\left(\mathbf{U I} \mathbf{S}_{1}, \mathbf{S}_{2}\right) \mid \mathbf{S}_{2}\right)=\mathrm{E}\left(\mathbf{U} \mid \mathbf{S}_{2}\right)=\mathrm{a}_{2}\left(\mathbf{S}_{2}\right)+\mathrm{E}_{\mathrm{S} 1}\left(\mathrm{a}_{1}\left(\mathbf{S}_{1}\right) \mid \mathbf{S}_{2}\right)+\mathrm{C}
\end{aligned}
$$

et

$$
\mathrm{E}\left(\mathbf{U} \mid \mathbf{S}_{1}\right)+\mathrm{E}\left(\mathbf{U} \mid \mathbf{S}_{2}\right)=\mathrm{E}\left(\mathbf{U} \mid \mathbf{S}_{1}, \mathbf{S}_{2}\right)+\mathrm{E}_{\mathrm{S} 1}\left(\mathrm{a}_{1}\left(\mathbf{S}_{1}\right) \mid \mathbf{S}_{2}\right)+\mathrm{E}_{\mathrm{S} 2}\left(\mathrm{a}_{2}\left(\mathbf{S}_{2}\right) \mid \mathbf{S}_{1}\right)+\mathrm{C},
$$

mais à présent les termes $\mathrm{E}_{\mathrm{S} 1}\left(\mathrm{a}_{1}\left(\mathbf{S}_{1}\right) \mid \mathbf{S}_{2}\right)$ et $\mathrm{E}_{\mathrm{S} 2}\left(\mathrm{a}_{2}\left(\mathbf{S}_{2}\right) \mid \mathbf{S}_{1}\right)$ ne se réduisent pas respectivement à $\mathrm{E}_{\mathrm{S} 1}\left(\mathrm{a}_{1}\left(\mathbf{S}_{1}\right)\right)$ et $\mathrm{E}_{\mathrm{S} 2}\left(\mathrm{a}_{2}\left(\mathbf{S}_{2}\right)\right)$ comme dans le Théorème 2.

Il en résulte la relation

$$
\mathrm{E}\left(\mathbf{U} \mid \mathbf{S}_{1}, \mathbf{S}_{2}\right)=\mathrm{E}\left(\mathbf{U} \mid \mathbf{S}_{1}\right)-\mathrm{E}_{\mathrm{S} 2}\left(\mathrm{a}_{2}\left(\mathbf{S}_{2}\right) \mid \mathbf{S}_{1}\right)+\mathrm{E}\left(\mathbf{U} \mid \mathbf{S}_{2}\right)-\mathrm{E}_{\mathrm{S} 1}\left(\mathrm{a}_{1}\left(\mathbf{S}_{1}\right) \mid \mathbf{S}_{2}\right)-\mathrm{C},
$$

c'est une autre expression du développement et elle conduit à l'identification terme à terme

$$
\begin{aligned}
& \mathrm{a}_{1}\left(\mathbf{S}_{1}\right)=\mathrm{E}\left(\mathbf{U} \mid \mathbf{S}_{1}\right)-\mathrm{E}_{\mathrm{S} 2}\left(\mathrm{a}_{2}\left(\mathbf{S}_{2}\right) \mid \mathbf{S}_{1}\right), \\
& \mathrm{a}_{2}\left(\mathbf{S}_{2}\right)=\mathrm{E}\left(\mathbf{U} \mid \mathbf{S}_{2}\right)-\mathrm{E}_{\mathrm{S} 1}\left(\mathrm{a}_{1}\left(\mathbf{S}_{1}\right) \mid \mathbf{S}_{2}\right),
\end{aligned}
$$


$\mathrm{C}=-\mathrm{C}=0$.

On obtient ainsi un système fonctionnel que les deux applications $a_{1}$ et $a_{2}$ d'un développement linéaire à variable séparées doivent nécessairement vérifier.

On vérifie que ce résultat contient le cas particulier de l'indépendance, et dans le cas général on en déduit un résultat d'impossibilité :

COROLLAIRE. Hormis le cas où les réponses univariées $\mathrm{E}\left(\mathbf{U} \mid \mathbf{S}_{1}\right)$ et $\mathrm{E}\left(\mathbf{U} \mid \mathbf{S}_{2}\right)$ sont constantes, $\mathrm{a}_{1}\left(\mathbf{S}_{1}\right)=\mathrm{E}\left(\mathbf{U} \mid \mathbf{S}_{1}\right)-\mathrm{C}_{1}$ et $\mathrm{a}_{2}\left(\mathbf{S}_{2}\right)=\mathrm{E}\left(\mathbf{U} \mid \mathbf{S}_{2}\right)-\mathrm{C}_{2}$ ne peuvent être des solutions dans le cas général de la dépendance.

En effet $\mathrm{E}_{\mathrm{S} 2}\left(\mathrm{E}\left(\mathbf{U} \mid \mathbf{S}_{2}\right) \mid \mathbf{S}_{1}\right)$ et $\mathrm{E}_{\mathrm{S} 1}\left(\mathrm{E}\left(\mathbf{U} \mid \mathbf{S}_{1}\right) \mid \mathbf{S}_{2}\right)$ ne peuvent être constants dans le cas de la dépendance de la loi conjointe du plan $\left(\mathbf{S}_{1}, \mathbf{S}_{2}\right)$ lorsque ni $\mathrm{E}\left(\mathbf{U} \mid \mathbf{S}_{1}\right)$ ni $\mathrm{E}\left(\mathbf{U} \mid \mathbf{S}_{2}\right)$ ne sont eux-mêmes constants.

\subsection{UNE POURSUITE ÉVENTUELLE AVEC LA DÉPENDANCE}

\subsubsection{Un premier bilan}

La résolution du système du Théorème 3 n'est pas aisée, et dans le cas plus simple de valeurs discrètes pour les variables de conditionnement $s_{1 i}$ et $s_{2 k}$ on n'a pas de solution dans le cas général [Maurin, 2007]. Un terme d'interaction

$$
\mathrm{c}_{\mathrm{dep}}\left(\mathbf{S}_{1}, \mathbf{S}_{2}\right)=\mathrm{E}\left(\mathbf{U} \mid \mathbf{S}_{1}, \mathbf{S}_{2}\right)-\mathrm{a}_{1}\left(\mathbf{S}_{1}\right)-\mathrm{a}_{2}\left(\mathbf{S}_{2}\right)-\mathrm{C} \quad\left[\mathrm{c}_{\mathrm{dep}}\right]
$$

analogue à celui de l'indépendance du $\S 6.3 .1$ n'est donc guère envisageable dans le cas général, faute de terme de référence $d_{\text {ref,dep }}$ identifiable ; et même s'il en était ainsi les applications $a_{1}\left(\mathbf{S}_{1}\right)$ et $a_{2}\left(\mathbf{S}_{2}\right)$ n'auraient pas la même interprétation simple que celle de $\mathrm{E}\left(\mathbf{U} \mid \mathbf{S}_{1}\right)$ et $\mathrm{E}\left(\mathbf{U} \mid \mathbf{S}_{2}\right)$ avec les réponses simplement univariées. Cela n'empêche nullement cependant que $c_{\text {dep }}$ possède des propriétés formelles communes avec celles de $c_{\text {indep}}$, (Annexe 5).

Cela signifie que la définition [cg] n'est pleinement justifiée que dans le cas de l'indépendance de la loi conjointe de $\left(\mathbf{S}_{1}, \mathbf{S}_{2}\right)$, moyennant quoi dans le cas général, on peut s'y méprendre et passer outre [Maurin, 2001]. On se trouve aussi dans une situation plus ou moins analogue à celle que l'on rencontre dans les plans d'expériences multivariés avec interaction. Dans cet autre contexte en effet l'interaction est commode à définir et à tester lorsque les plans sont complets avec un même nombre de répétitions d'épreuves par cases, et beaucoup moins commode dans les autres cas [Chapouille, 1973]. À la suite de quoi on peut ici introduire la distinction entre l'interaction véritable $\mathrm{c}_{\text {indep }}$ lorsque la loi de $\left(\mathbf{S}_{1}, \mathbf{S}_{2}\right)$ est indépendante, et l'interaction simplement apparente que donne la définition $[\mathrm{cg}]$ dans les autres cas.

\subsubsection{L'interaction à son tour décomposée}

On peut néanmoins appliquer à l'interaction générale $\mathrm{c}\left(\mathbf{S}_{1}, \mathbf{S}_{2}\right)$ de $[\mathrm{cg}]$ la même définition [cg] pour introduire sa propre interaction

$$
\operatorname{cc}\left(\mathbf{S}_{1}, \mathbf{S}_{2}\right)=\mathrm{c}\left(\mathbf{S}_{1}, \mathbf{S}_{2}\right)-\mathrm{c}_{1}\left(\mathbf{S}_{1}\right)-\mathrm{c}_{2}\left(\mathbf{S}_{2}\right)+\mathrm{E}(\mathbf{c})
$$

à partir des espérances conditionnelles $c_{1}\left(\mathbf{S}_{1}\right)$ et $c_{2}\left(\mathbf{S}_{2}\right)$ du Lemme 1 , en sachant que $\mathrm{E}(\mathbf{c})=0$. Cela définit « l'interaction de l'interaction »; elle se réduit identiquement à $\mathbf{c}$ dans le cas de l'indépendance puisque $\mathbf{c}_{1}$ et $\mathbf{c}_{2}$ sont identiquement nuls dans ce cas 
(Lemme 3), et cela permet d'écrire dans le cas général $\mathrm{c}\left(\mathbf{S}_{1}, \mathbf{S}_{2}\right)=\mathrm{c}_{1}\left(\mathbf{S}_{1}\right)+\mathrm{c}_{2}\left(\mathbf{S}_{2}\right)+$ $\operatorname{cc}\left(\mathbf{S}_{1}, \mathbf{S}_{2}\right)$.

THÉORÈME 4. On a la décomposition algébrique $\operatorname{var}(\mathbf{c})=\operatorname{var}\left(\mathbf{c}_{1}-\mathbf{c}_{2}\right)+\operatorname{var}(\mathbf{c c})$.

En effet

$$
\operatorname{var}(\mathbf{c})=\operatorname{var}\left(\mathbf{c}_{1}\right)+\operatorname{var}\left(\mathbf{c}_{2}\right)+\operatorname{var}(\mathbf{c c})+2 \operatorname{cov}\left(\mathbf{c}_{1}, \mathbf{c}_{2}\right)+2 \operatorname{cov}\left(\mathbf{c}_{1}, \mathbf{c c}\right)+2 \operatorname{cov}\left(\mathbf{c}_{2}, \mathbf{c c}\right) .
$$

a) on a par exemple

$$
\operatorname{cov}\left(\mathbf{c}_{1}, \mathbf{c c}\right)=\operatorname{cov}\left(\mathbf{c}_{1}, \mathbf{c}-\mathbf{c}_{1}-\mathbf{c}_{2}\right)=-\operatorname{cov}\left(\mathbf{c}_{1}, \mathbf{c}_{2}\right)+\operatorname{cov}\left(\mathbf{c}_{1}, \mathbf{c}-\mathbf{c}_{1}\right)
$$

avec

$$
\begin{array}{ll} 
& \mathbf{c}=\mathbf{U}_{12}-\mathbf{U}_{1}-\mathbf{U}_{2}+\mathrm{E}(\mathbf{U}), \\
& \mathbf{c}_{1}=\mathrm{E}(\mathbf{U})-\mathrm{E}_{\mathrm{S} 2}\left(\mathbf{U}_{2} \mid \mathbf{S}_{1}\right), \mathbf{c}_{2}=\mathrm{E}(\mathbf{U})-\mathrm{E}_{\mathrm{S} 1}\left(\mathbf{U}_{1} \mid \mathbf{S}_{2}\right), \\
\text { et } \quad & \mathbf{c}-\mathbf{c}_{1}=\mathbf{U}_{12}-\mathbf{U}_{1}-\mathbf{U}_{2}+\mathrm{E}_{\mathrm{S} 2}\left(\mathbf{U}_{2} \mid \mathbf{S}_{1}\right) .
\end{array}
$$

Les deux termes $\mathbf{c}_{1}, \mathbf{c}-\mathbf{c}_{1}$ sont centrés et l'on a

$$
\begin{aligned}
& \operatorname{cov}\left(\mathbf{c}_{1}, \mathbf{c}-\mathbf{c}_{1}\right)=\mathrm{E}_{\mathrm{S} 1}\left[\mathrm{E}_{\mathrm{S} 2}\left\{\left(\mathrm{E}(\mathbf{U})-\mathrm{E}_{\mathrm{S} 2}\left(\mathbf{U}_{2} \mid \mathbf{S}_{1}\right)\right) .\left(\mathbf{U}_{12}-\mathbf{U}_{1}-\mathbf{U}_{2}+\mathrm{E}_{\mathrm{S} 2}\left(\mathbf{U}_{2} \mid \mathbf{S}_{1}\right)\right) \mid \mathbf{S}_{1}\right\}\right] \\
& =\mathrm{E}_{\mathrm{S} 1}\left[\left(\mathrm{E}(\mathbf{U})-\mathrm{E}_{\mathrm{S} 2}\left(\mathbf{U}_{2} \mid \mathbf{S}_{1}\right)\right) . \mathrm{E}_{\mathrm{S} 2}\left\{\left(\mathbf{U}_{12}-\mathbf{U}_{1}-\mathbf{U}_{2}+\mathrm{E}_{\mathrm{S} 2}\left(\mathbf{U}_{2} \mid \mathbf{S}_{1}\right)\right) \mid \mathbf{S}_{1}\right\}\right] .
\end{aligned}
$$

L'espérance $\mathrm{E}_{\mathrm{S} 2}\left\{\left(\mathbf{U}_{12}-\mathbf{U}_{1}-\mathbf{U}_{2}+\mathrm{E}_{\mathrm{S} 2}\left(\mathbf{U}_{2} \mid \mathbf{S}_{1}\right)\right) \mid \mathbf{S}_{1}\right\}$ est nulle puisque $\mathrm{E}_{\mathrm{S} 2}\left(\mathbf{U}_{12} \mid \mathbf{S}_{1}\right)=\mathbf{U}_{1}$, et l'on a $\operatorname{cov}\left(\mathbf{c}_{1}, \mathbf{c}-\mathbf{c}_{1}\right)=0$ et $\operatorname{cov}\left(\mathbf{c}_{1}, \mathbf{c c}\right)=-\operatorname{cov}\left(\mathbf{c}_{1}, \mathbf{c}_{2}\right)$.

b) on a l'équivalent pour $\operatorname{cov}\left(\mathbf{c}_{2}, \mathbf{c c}\right)$;

c) il en résulte que $\operatorname{cov}\left(\mathbf{c}_{1}, \mathbf{c c}\right)+\operatorname{cov}\left(\mathbf{c}_{2}, \mathbf{c c}\right)=-2 \operatorname{cov}\left(\mathbf{c}_{1}, \mathbf{c}_{2}\right)$,

et finalement que $\operatorname{var}(\mathbf{c})=\operatorname{var}\left(\mathbf{c}_{1}\right)+\operatorname{var}\left(\mathbf{c}_{2}\right)-2 \operatorname{cov}\left(\mathbf{c}_{1}, \mathbf{c}_{2}\right)+\operatorname{var}(\mathbf{c c})$.

THÉORÈME 5. $\operatorname{var}\left(\mathbf{c}_{1}-\mathbf{c}_{2}\right)$ est nul si et seulement si la distribution du plan $\left(\mathbf{S}_{1}, \mathbf{S}_{2}\right)$ est indépendante.

En effet sous l'indépendance $\mathbf{c}_{1}$ et $\mathbf{c}_{2}$ sont nuls.

Réciproquement si $\operatorname{var}\left(\mathbf{c}_{1}-\mathbf{c}_{2}\right)=0, \mathbf{c}_{1}-\mathbf{c}_{2}$ est une constante, cela entraîne que $\mathbf{c}_{1}$ et $\mathbf{c}_{2}$ sont individuellement constants en fonction de leur argument respectif, et donc que les distributions conditionnelles $\mathbf{S}_{2} \mid \mathbf{S}_{1}$ sont identiques, de même que les distributions conditionnelles $\mathbf{S}_{1} \mid \mathbf{S}_{2}$.

\subsubsection{Un bilan mitigé et inégal}

À l'issue de cet examen, on remarque en premier que de manière générale les expressions théoriques comme $\mathrm{E}\left(\mathbf{U} \mid \mathbf{S}_{1}, \mathbf{S}_{2}\right), \mathrm{c}\left(\mathbf{S}_{1}, \mathbf{S}_{2}\right)$ et les espérances conditionnelles sont de portée générale, tandis que la connaissance des valeurs numériques donne une information simplement locale. De son côté la variance de la réponse conjointe, Théorème 1 et Annexe 4, apporte une information de niveau global sur l'importance de l'interaction, comme le font en d'autres circonstances statistiques un khi2 de tableau de contingence ou un rapport de corrélation.

Dans le cas de l'indépendance, l'interaction de l'interaction apporte à sa manière un complément au niveau global de la variance puisqu'elle permet d'identifier un nouveau terme $\operatorname{var}\left(\mathbf{c}_{1}-\mathbf{c}_{2}\right)$ qui est uniquement dû à la dépendance du plan $\left(\mathbf{S}_{1}, \mathbf{S}_{2}\right)$, et son complémentaire $\operatorname{var}(\mathbf{c c})$ qui ne s'annule pas sous l'indépendance. Ce dernier 
pourrait ainsi mieux représenter la contribution de l'interaction véritable dans les décompositions de $\operatorname{var}(\mathbf{c})$ et de $\operatorname{var}\left(\mathbf{U}_{12}\right)=\operatorname{var}\left(\mathbf{U}_{1}-\mathbf{U}_{2}\right)+\operatorname{var}\left(\mathbf{c}_{1}-\mathbf{c}_{2}\right)+\operatorname{var}(\mathbf{c c})$. Il en résulte que si l'on ne peut expliciter la part de l'interaction véritable sous forme locale dans le cas général, on peut s'en approcher sur le plan global avec la décomposition des variances.

Cependant on peut s'attendre à ce que ces théorèmes se répètent pour l'interaction de cc, et donc que var(cc) ne soit pas la partie de $\operatorname{var}(\mathbf{U})$ qui corresponde à l'interaction véritable globale.

\subsection{DISCUSSION ET CONCLUSION}

On a noté combien les intervalles successifs s'adaptent très simplement au contexte des situations multidimensionnelles. Ils n'ont que les axiomes d'Adams et Messick à respecter $(\$ 2.3)$, et la réponse conjointe $\mathrm{E}\left(\mathbf{U} \mid \mathbf{S}_{1}, \mathbf{S}_{2}\right)$ qui en résulte est à même de révéler des variations en dehors de tout schéma fonctionnel particulier (§ 4.5).

Cette réponse donne lieu par ailleurs à deux lectures complémentaires. La première concerne la réponse proprement dite, ses diverses coupes partielles univariées (§ 4.1), ainsi que les réponses univariées qui proviennent (ou proviendraient) d'un recueil allégé simplement univarié grâce aux propriétés des espérances conditionnelles (§ 4.4). Et tout cela sous le double aspect d'une connaissance globale et locale.

La seconde lecture se produit au niveau de l'interaction entre les variables ( $\$ 6)$. Si la forme de cette dernière est naturellement connue lorsque les réponses suivent un schéma fonctionnel donné, il s'avère que dans le cadre des intervalles successifs, elle est elle-même révélée par le mesurage au même titre que la réponse conjointe. C'est un apport supplémentaire intéressant qui est à mettre au crédit des intervalles successifs. Il n'est cependant pas porteur de la même information dans tous les cas. Le cas de l'indépendance de la distribution des variables d'exposition dans le plan d'expérience de $\left(\mathbf{S}_{1}, \mathbf{S}_{2}\right)$ est le plus favorable puisqu'il permet d'expliciter complètement l'interaction entre les variables ainsi que sa contribution dans la variance totale ; on a donc intérêt sur le plan pratique à le privilégier dans la mesure du possible.

\section{DES EXEMPLES BIVARIES}

\subsection{L'INCONFORT GESTUEL DE $§ 3.2$, SUITE}

Les situations de l'enquête dépendent de trois variables descriptives géométriques, et l'on dispose de relevés assez riches pour obtenir des pavés de contingence importants. Ici nous étudions la réponse en fonction de l'orientation $\mathbf{S}_{1}$ et la distance de la cible à atteindre $\mathbf{S}_{2}$, avec 5 valeurs d'orientation et 4 valeurs de distance ; toutes les cases du plan $\mathbf{S}_{1} \mathbf{S}_{2}$ sont considérées et les données sont mises sous forme de tableau de contingence à 20 lignes et 5 colonnes, (Tableau 6). 


\begin{tabular}{|c|c|c|c|c|c|}
\hline \multicolumn{2}{|c|}{$s_{1 i} s_{2 k}$} & \multicolumn{4}{|c|}{$C_{j}, j=1 \ldots 5$} \\
\hline 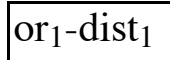 & 55 & 20 & 5 & 0 & 0 \\
\hline or $_{1}$-dist 2 & 59 & 73 & 22 & 5 & 1 \\
\hline or $_{1}$-dist 3 & 44 & 84 & 61 & 10 & 1 \\
\hline or $_{1}$-dist 4 & 3 & 62 & 96 & 34 & 5 \\
\hline or $_{2}$-dist 1 & 59 & 19 & 2 & 0 & 0 \\
\hline or $_{2}-$ dist $_{2}$ & 120 & 61 & 15 & 4 & 0 \\
\hline or $_{2}-$ dist $_{3}$ & 60 & 93 & 42 & 5 & 0 \\
\hline or $_{2}$-dist 4 & 17 & 65 & 85 & 31 & 2 \\
\hline or $_{3}$-dist 1 & 48 & 26 & 6 & 0 & 0 \\
\hline or $_{3}$-dist 2 & 91 & 71 & 35 & 3 & 0 \\
\hline or $_{3}-$ dist $_{3}$ & 35 & 89 & 67 & 9 & 0 \\
\hline $\mathrm{or}_{3}$-dist 4 & 9 & 43 & 101 & 42 & 5 \\
\hline or $_{4}$-dist 1 & 22 & 33 & 20 & 3 & 2 \\
\hline or $_{4}$-dist 2 & 41 & 91 & 49 & 11 & 8 \\
\hline or $_{4}$-dist 3 & 25 & 75 & 79 & 15 & 6 \\
\hline or $_{4}$-dist 4 & 1 & 50 & 90 & 47 & 12 \\
\hline or $_{5}$-dist 1 & 6 & 6 & 25 & 22 & 21 \\
\hline or $_{5}$-dist 2 & 7 & 38 & 66 & 42 & 47 \\
\hline or $_{5}-$ dist $_{3}$ & 3 & 33 & 72 & 44 & 48 \\
\hline or $_{5}$-dist 4 & 0 & 9 & 65 & 66 & 60 \\
\hline
\end{tabular}

TABLEAU 6. Les réponses en fonction de l'orientation et de la distance

Les conditions $\left[\mathrm{C}_{\mathrm{AM}}\right]$ sont correctement vérifiées et la minimisation du stress avec la loi normale donne les valeurs conjointes :

\begin{tabular}{l|cccc|} 
& dist $_{1}$ & dist $_{2}$ & dist $_{3}$ & dist $_{4}$ \\
\cline { 2 - 5 } or $_{1}$ & 1,000 & 6,019 & 8,919 & 13,911 \\
or $_{2}$ & 0,911 & 2,058 & 7,250 & 11,956 \\
or $_{3}$ & 2,792 & 5,337 & 9,367 & 13,695 \\
or $_{4}$ & 8,128 & 9,389 & 11,155 & 15,437 \\
or $_{5}$ & 18,394 & 17,255 & 17,798 & 20,000 \\
\cline { 2 - 5 }
\end{tabular}

TABLEAU 7. Les mesurages numériques $\mathrm{u}_{\mathrm{ik}}$ orientation - distance puis les correspondances univariées sur-sommées :

- en fonction de l'orientation, $\mathrm{u}_{1 \mathrm{i}}=\mathrm{E}\left(\mathbf{U} \mid \mathbf{S}_{1}=\mathrm{s}_{1 \mathrm{i}}\right), \mathrm{u}_{2 \mathrm{k}}=\mathrm{E}\left(\mathbf{U} \mid \mathbf{S}_{2}=\mathrm{s}_{2 \mathrm{k}}\right)$

$$
\mathrm{u}_{1 \mathrm{i}}: \quad 8,764 \quad 6,361 \quad 8,681 \quad 11,539 \quad 18,356
$$

- en fonction de la distance

$$
\mathrm{u}_{2 \mathrm{k}}: \quad 6,245 \quad 8,095 \quad 10,898 \quad 15,000
$$

et l'espérance $\mathrm{E}(\mathbf{U})=10,764$. 
On peut figurer ces deux lois univariées dans un même graphe avec l'ordonnée commune pour la réponse, et des modalités qualitatives des variables en abscisse, Figure 8 (on retrouve pour l'orientation la correspondance univariée de la Figure 5). Les étendues respectives sont $\Delta \mathrm{u}_{\mathrm{or}}=11,99$ et $\Delta \mathrm{u}_{\mathrm{dist}}=8,755$, avec un ratio de 1,37 en faveur de l'orientation.

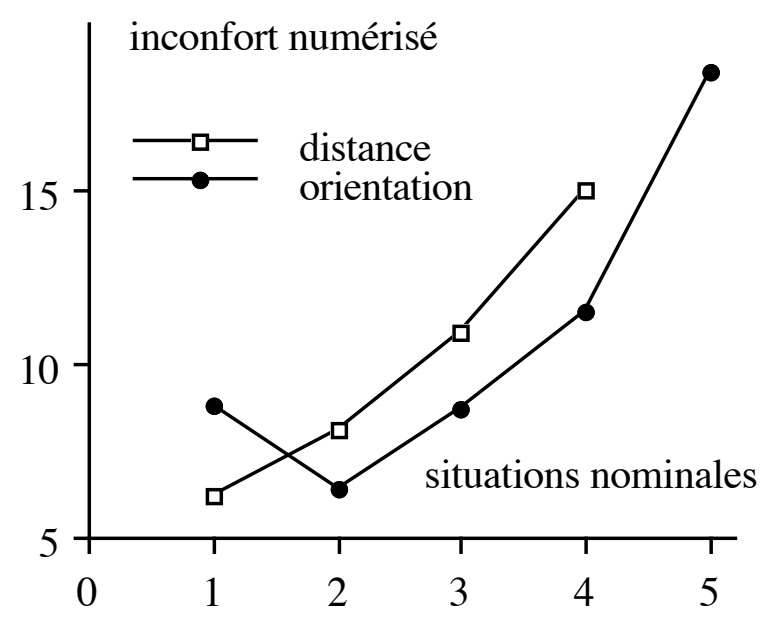

FIGURE 8. Lois univariées de l'inconfort en fonction de la distance et de l'orientation

En outre le plan d'expérience entre les variables est ici très proche d'une distribution d'indépendance (Tableau 8 , avec une seule case qui ne respecte pas l'indépendance, en italique),

\begin{tabular}{l|cccc|}
\multicolumn{1}{c}{} & dist $_{1}$ & dist $_{2}$ & dist $_{3}$ & dist $_{4}$ \\
\cline { 2 - 5 } or $_{1}$ & 20 & 40 & 50 & 50 \\
or $_{2}$ & 20 & 50 & 50 & 50 \\
or $_{3}$ & 20 & 50 & 50 & 50 \\
or $_{4}$ & 20 & 50 & 50 & 50 \\
or $_{5}$ & 20 & 50 & 50 & 50 \\
\cline { 2 - 5 } & & &
\end{tabular}

TABLEAU 8. Le plan d'expérience orientation - distance

on peut donc raisonnablement considérer que les conditions sont réunies pour que le terme c ne soit dû qu'à la seule interaction entre les variables, Tableau 9 :

\begin{tabular}{l|cccc|}
\multicolumn{1}{c}{} & dist $_{1}$ & dist $_{2}$ & dist $_{3}$ & dist $_{4}$ \\
\cline { 2 - 5 } or $_{1}$ & $\mathbf{- 3 , 2 4 5}$ & $-0,076$ & 0,0209 & 0,9109 \\
or $_{2}$ & $-0,931$ & $\mathbf{- 1 , 6 3 4}$ & 0,7547 & $\mathbf{1 , 3 5 8 7}$ \\
or $_{3}$ & $\mathbf{- 1 , 3 7}$ & $-0,675$ & 0,5519 & 0,7779 \\
or $_{4}$ & $\mathbf{1 , 1 0 7 9}$ & 0,5193 & $-0,518$ & $-0,338$ \\
or $_{5}$ & $\mathbf{4 , 5 5 6 8}$ & $\mathbf{1 , 5 6 8 1}$ & $-0,692$ & $\mathbf{- 2 , 5 9 2}$ \\
\hline
\end{tabular}

TABLEAU 9. Le terme d'interaction $\mathrm{c}_{\mathrm{ik}}$ orientation - distance 
Les variations univariées sur-sommées :

$$
\begin{array}{lccccc}
\mathrm{c}_{1 \mathrm{i}}: & -0,133 & 0,032 & 0,032 & 0,032 & 0,032 \\
\mathrm{c}_{2 \mathrm{k}}: & 0,0235 & -0,059 & 0,0235 & 0,0235 &
\end{array}
$$

sont très proches de zéro comme il se doit (puisque l'on est «proche » de l'indépendance).

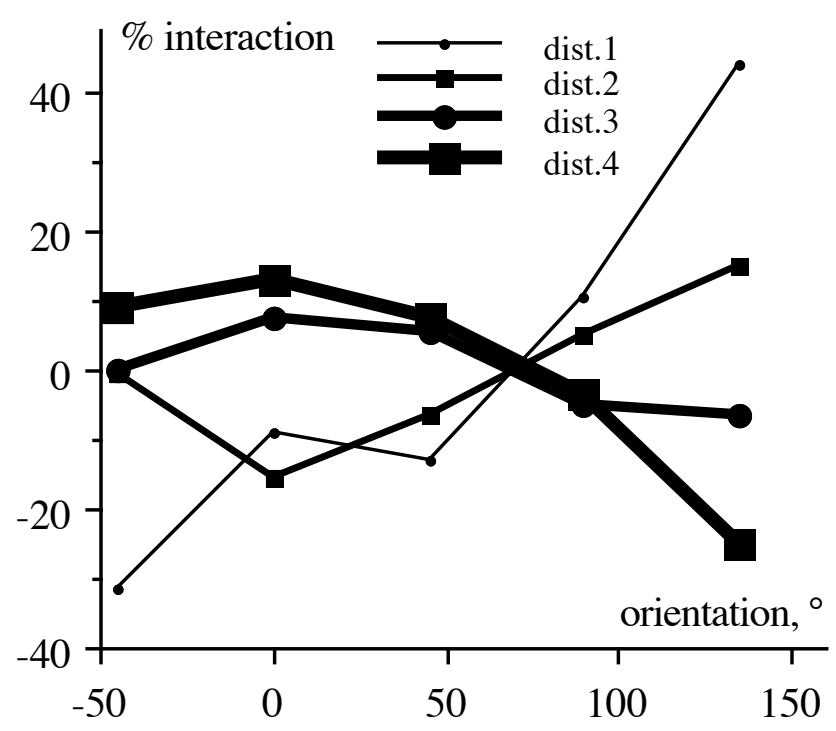

FIGURE 9. Les interactions par orientation et distance, en valeur relative (cf. Tableau 9)

Au titre des interprétations techniques hors de toute thématique on peut noter que :

- le signe de l'interaction est régulièrement disposé avec un bloc diagonal négatif pour une interaction inhibitrice quand on associe les valeurs extrémales pour les deux stimulations (en grisé), et un second bloc diagonal positif d'interaction amplificatrice ;

- les plus fortes valeurs absolues de l'interaction se situent plus en périphérie du plan qu'au centre, et les valeurs de c qui dépassent les $10 \%$ de la moyenne des étendues des lois univariées $\left(\Delta \mathrm{u}_{\text {or }}+\Delta \mathrm{u}_{\text {dist }}\right) / 2=10,375$ sont inscrites en gras dans le Tableau 9 ;

- la variance des $c_{i k}$ (la véritable interaction sur ce plan avec indépendance) s'élève à $6,5 \%$ de la variance des $u_{i k}$; l'interaction est ici assez faible. Figure 9.

Les valeurs relatives de $c_{i k}$ par rapport à $\left(\Delta \mathrm{u}_{\mathrm{or}}+\Delta \mathrm{u}_{\mathrm{dist}}\right) / 2$ sont indiquées en

\subsection{LA BI-EXPOSITION DE $§ 5$, SUITE}

Avec les $\mathrm{E}\left(\mathbf{U} \mid \mathbf{S}_{1}, \mathbf{S}_{2}\right), \mathrm{E}\left(\mathbf{U} \mid \mathbf{S}_{1}\right)$ et $\mathrm{E}\left(\mathbf{U} \mid \mathbf{S}_{2}\right)$ précédents on peut poursuivre et calculer l'interaction suivante (cf. Tableau 10) 


\begin{tabular}{l|ccccc|}
\multicolumn{1}{c}{} & $\mathrm{s}_{21}$ & $\mathrm{~s}_{22}$ & $\mathrm{~s}_{23}$ & $\mathrm{~s}_{24}$ & $\mathrm{~s}_{25}$ \\
\cline { 2 - 6 } $\mathrm{s}_{11}$ & & $\mathbf{3 , 1 5 5}$ & 0,271 & & \\
$\mathrm{~s}_{12}$ & $-1,825$ & $\mathbf{4 , 2 8 0}$ & $\mathbf{3 , 5 9 1}$ & $-2,346$ & \\
$\mathrm{~s}_{13}$ & 2,524 & $-0,450$ & 2,451 & 0,419 & $\mathbf{- 5 , 2 0 7}$ \\
$\mathrm{s}_{14}$ & 0,021 & $-0,814$ & $-0,986$ & 1,682 & 2,498 \\
$\mathrm{~s}_{15}$ & & & $\mathbf{- 4 , 6 6 6}$ & $\mathbf{- 4 , 3 1 9}$ & $\mathbf{- 3 , 2 7 1}$ \\
\cline { 3 - 6 } & & & & &
\end{tabular}

TABLEAU 10. Le terme d'interaction $\mathrm{c}_{\mathrm{ik}}$ de la bi-exposition acoustique

Cependant la distribution conjointe du plan expérimental, avec notamment des cases vides, n'est pas une distribution indépendante (cf. Tableau 4), et il ne peut s'agir ici que de l'interaction apparente. Il en résulte que les variations par sur-sommation ne sont pas identiquement nulles

$$
\begin{array}{lllllll}
\mathrm{c}_{1 \mathrm{i}}: & & 0,263 & 1,186 & 0,830 & -0,792 & -1,845 \\
\mathrm{c}_{2 \mathrm{k}}: & & 1,537 & 2,090 & 0,396 & 0,533 & -3,886
\end{array}
$$

et que les commentaires que l'on peut en faire sont plutôt mal avisés (comme en fin de [Maurin, 2001]). On en retire néanmoins des enseignements en amont de toute thématique particulière :

- le tableau des $c_{i k}$ montre une certaine régularité avec une disposition en bandes diagonales du signe ;

- par rapport à la valeur de référence $\left(\Delta \mathrm{u}_{\text {rail }}+\Delta \mathrm{u}_{\text {rou }}\right) / 2=11,69$ il y a 11 interactions en valeurs absolues (sur 19, soit $58 \%$ ) qui dépassent $20 \%$; la plus élevée $(5,207$ ) atteint $44,5 \%$, celles qui sont à plus de $25 \%$ figurent en gras dans le Tableau 10 ;

- la variance des $c_{i k} s$ 'élève à $26,6 \%$ de la variance des $u_{i k}$, et celle des $\mathrm{cc}_{\mathrm{ik}}$ à $15,9 \%$; sur ces données l'interaction que l'on observe est assez élevée.

\subsection{DES LOIS DE RÉPONSE PAR SOUS-POPULATIONS}

Il y a un cas multivarié particulier lorsque l'un des deux arguments $\mathbf{S}_{2}$ est un facteur qualitatif $\mathbf{P}$ comme un identifiant de sous-population, (age, sexe, CSP, ...). Les coupes partielles de la solution conjointe $(\$ 4.1)$ sont les lois de réponse de chaque souspopulation, et l'on peut encore définir ici aussi l'interaction entre $\mathbf{S}_{1}$ et $\mathbf{P}$, d'autant mieux que le plan des deux variables $\left(\mathbf{S}_{1}, \mathbf{P}\right)$ est indépendant [Maurin, 2007].

\section{CONCLUSIONS}

La numérisation, et notamment celle de la réponse de l'homme, est un dessein qui concerne plusieurs disciplines ; il s'agit par exemple de l'utilité en économie, de la gêne à la suite d'une exposition en environnement, du confort ou de l'inconfort en ergonomie, de la sensation en psychophysique, sans compter tout ce qui concerne $L a$ mesure de l'esprit avec toutes les échelles qui, depuis Binet, s'appliquent à mesurer l'intelligence [Martin, 1997]. Sous l'effet du quantitative imperative signalé par Michell, cela peut notamment se prêter à un certain number appeal récurrent [Maurin, 2003(b)], et pour y répondre de nombreuses méthodes de saisie d'une part et de traitement de l'autre ont été introduites. Parmi elles, la théorie du mesurage, en date du 
$\mathrm{XX}^{\mathrm{e}}$, repose sur une véritable réflexion à propos de la mesure des phénomènes ; c'est sans doute le cadre technique et formel le plus satisfaisant, à l'abri d'un postulat implicite sur l'existence d'un correspondant numérique comme c'est souvent le cas autrement.

D'un autre côté la pratique du recueil de réponses subjectives avec des échelles de catégories ordonnées est bien répandue. Les intervalles successifs d'Adams et Messick constituent un prolongement de la loi des jugements catégoriels de Saffir et Thurstone dans le cadre du mesurage, et à ce titre ils concernent tous les stimuli thurstoniens qui résultent de l'élargissement envisagé par Thurstone vis-à-vis des stimuli susceptibles d'être comparés [Martin, 1997]. Cette méthode répond au souci relationnel et numérique de la psychophysique avec des stimulations univariées, elle s'étend aisément au cas des situations multivariées, et elle y présente une bien plus grande souplesse d'utilisation qu'avec les techniques plus habituelles du mesurage conjoint. Pour deux variables comme dans le cas présent, les intervalles successifs permettent en effet d'aller au-delà de la simple additivité avec la présence d'un terme d'interaction libre de tout modèle dans la réponse globale. Ils évitent ainsi de devoir se soucier de divers axiomes structurels comme ceux de cancellation ou de decomposability [Bouyssou, Pirlot, 2002 ; Michell, 1990 ; Roberts, 1979], et plus encore dans le cas de l'indépendance des plans d'expérience.

Les intervalles successifs s'appliquent à tout stimulus thurstonien, et lorsque pour l'un d'entre eux on est tenté par la rhétorique du nombre et des lois de réponse numériques associées, leur mise en oeuvre se présente comme une heuristique pertinente et commode, voire comme une certaine maïeutique relationnelle et quantitative. Et si, au moment de terminer, on veut rappeler la présence du volet artistique qu'Annett, Keeney et consorts ne manquent pas d'évoquer, il se pourrait que celui-ci réside dans de nouveaux modes de gestion plus originaux qu'à l'ordinaire des impératifs mathématiques et scientifiques.

Remerciements. L'auteur exprime sa reconnaissance envers les rapporteurs pour leur lecture soignée, et pour les propositions d'améliorations, de simplifications et d'enrichissements qu'ils ont signalées.

\section{BIBLIOGRAPHIE}

ABRAMOWITZ M., STEGUN I., Handbook of mathematical functions, Dover, 1970.

ADAMS E., MESSICK S., “An axiomatic formulation and generalisation of successive intervals scaling”, Psychometrika 23, n 4, 1958, p. 355-368.

ANNETT J., “Subjective rating scales: science or art?”, Egonomics, vol 45 n 14, 2002, p. 966-987.

BEINAT E., Multiattribute value functions for environmental management, Tinbergen (The Netherlands), Tinbergen Institute Research Series, 1995.

BONNET C., Manuel pratique de psychophysique, Paris, A. Colin, 1986.

BOUDON R., L'analyse mathématique des faits sociaux, Paris, Plon, 1967.

BOUYSSOU D., PIRLOT M., "Nontransitive decomposable conjoint measurement", Journal of Math. Psych., vol 46, 2002, p. 677-703

CHAPOUILLE P., Planification et analyse des expériences, Paris, Masson \& Cie, 1973.

CHEVALOT N., WANG X., "An experimental investigation of the discomfort of arm reaching movements in a seated position", SAE International conference and exposition of digital human modelling for design and engineering, Oakand University, Rochester Michigan, USA, June, paper 200401-2194, 2004. 
DEBATY P., La mesure des attitudes, Paris, Presses Universitaires de France, 1967.

FALMAGNE J.-C., "Random conjoint measurement and loudness summation", Psychological review 83, $\mathrm{n}^{\circ} 1,1976$, p. 65-79.

FALMAGNE J.C., Elements of psychophysical theory, Oxford, Oxford University Press, 1985.

FELLER W., An introduction to probability theory and its applications, vol. II, Hoboken (NJ), J. Wiley and sons, 1971.

GELL-MANN M., Le quark et le jaguar, Paris, Flammarion, 1995.

HAMERLE A., TUTZ G., "Goodness of fit tests for probabilistic measurement models", J. of Math. Psychology 21, 1980, p. 153-167.

HAND D., Measurement theory and practice, the world through quantification, London, Arnold, 2004.

JOB R.F.S., HATFIELD J., «Responses to noise from combined sources and regulation against background noise levels", Proceedings of Inter-Noise-2001, La Haye, 2001.

KEENEY K.L., Value-focused thinking, Cambridge (MA), Harvard University Press, 1992.

LUCE R.D., GALANTER E., "Discrimination, Psychophysical scaling", in Luce, Bush, Galanter, Handbook of mathematical psychology, 193-243, 244-307, J. Wiley and sons, 1963.

LUCE R.D., TUCKEY J.W., "Simultaneous conjoint measurement, a new type of fundamental measurement”, Journal of Math. Psych, vol 1, 1964, p. 1-27.

MARTIN O., La mesure de l'esprit, Paris, L'Harmattan, 1997.

MAURIN M., « L'association du mesurage additif conjoint et des intervalles successifs », Mathématiques et Sciences humaines 96, 1986, p. 5-29.

MAURIN M., "The measurement of combined annoyances in multi-noise exposure", Proceedings of Inter-Noise-2001, La Haye, 2001.

MAURIN M., «Réflexions à propos des relations numériques entre le bruit des transports et la gêne exprimée », Recherche, Transports, Sécurité 78, 2003(a), p. 63-77.

MAURIN M., Measures and measurement from data, advanced mathematical basements, Young Researchers Seminar ECTRI-FERSI, INRETS-Lyon, 2003(b), [version française : La théorie du mesurage, INRETS, 2004].

MAURIN M., Les réponses par échelles de catégories ordonnées, leur traitement numérique et la méthode des intervalles successifs, tome 1 les situations unidimensionnées, rapport INRETS-LTE $\mathrm{n}^{\circ} 0635,2006$.

MAURIN M., Les réponses par échelles de catégories ordonnées, leur traitement numérique et la méthode des intervalles successifs, tome 2 les situations multidimensionnées, rapport INRETS-LTE $\mathrm{n}^{\circ} 0709,2007$.

MAURIN M., LAMBERT J., ALAUZET A., Enquête nationale sur le bruit des transports en France, INRETS, Rapport de Recherche n 71, 1988.

MICHELL J., An introduction to the logic of Psychological Measurement, Hillsdale (NJ), Lauwrence Erlbaum Associates Publishers, 1990.

PFANZAGL J., Theory of measurement, Würsburg, Physica Verlag, $2^{\text {nd }}$ revised version 1971.

ROBERTS F.J, Measurement theory, Reading (MA), Addison Wesley P. Co., 1979.

ROUSVAL B., Aide multicritère à l'évaluation de l'impact des transports sur l'environnement, Thèse de doctorat, Université Paris XI Dauphine, 2005.

ROY B., BOUYSSOU D., « Aide multicritère à la décision : méthodes de cas », Economica, 1993.

STEVENS S.S., "Issues in pychophysics, Psychological Review”, 78 n 5, 1971, p. 426-450.

SUPPES P., ZINNES J.L., "Basic measurement theory", Luce, Bush, Galanter, Handbook of math. psychology, vol 1, J. Wiley and sons, 1963, p. 1-76.

TORGERSON W.S., Theory and methods of scaling, New York, J. Wiley and sons, 1958. 


\section{ANNEXE 1 \\ LA NUMÉRISATION PONCTUELLE DES CATÉGORIES}

On a rappelé que l'option thurstonienne des intervalles successifs consiste à représenter des catégories qualitatives ordonnées par des intervalles sur $\mathrm{R}$, à l'opposé des pratiques plus répandues du scaling ou du scoring qui utilisent un scalaire unique. On peut cependant proposer une représentation scalaire des catégories qui respectent cette option en utilisant des espérances particulières des distributions de réponses par situation.

I - Pour chaque distribution conditionnée par rapport à $s_{i}$ et sur chaque intervalle $\left[t_{j-1}, t_{j}\right]$, la distribution des réponses de fonction de répartition $F_{i}(t)=F\left(\left(t-u_{i}\right) / \sigma_{i}\right)$ possède un accroissement $\Delta \mathrm{F}_{\mathrm{ij}}=\mathrm{F}\left(\frac{\mathrm{t}_{\mathrm{j}}-\mathrm{u}_{\mathrm{i}}}{\sigma_{\mathrm{i}}}\right)-\mathrm{F}\left(\frac{\mathrm{t}_{\mathrm{j}-1}-\mathrm{u}_{\mathrm{i}}}{\sigma_{\mathrm{i}}}\right)$. Il est naturel de résumer chaque intervalle par l'espérance de la distribution $F_{i}(t)$ tronquée $c_{j(i)}=\frac{1}{\Delta F_{i j}} \int_{t_{j}-1}{ }_{j} \frac{t}{\sigma_{i}} f\left(\frac{t-u_{i}}{\sigma_{i}}\right) d t=u_{i}+$ $\frac{\sigma_{i}}{\Delta F_{i i j}} \int_{b_{i, j-1}} b_{i, j} v f(v) d v$ avec le changement de variable $v=\left(t-u_{i}\right) / \sigma_{i}$, les nouvelles bornes d'intégration $b_{i, j}=\left(t_{j}-u_{i}\right) / \sigma_{i}$ pour $j=1 \ldots J-1$, et $b_{i, 0}=-\infty$ et $b_{i, J}=\infty$ dans le cas des extrêmes.

On a encore $c_{j(i)}=u_{i}+\sigma_{i} / \Delta F_{i j} K_{i, j}$ en posant les intégrales $K_{i, j}=\int_{b_{i, j-1}} b_{i, j} v f(v) d v$.

Les $\mathrm{c}_{\mathrm{j}(\mathrm{i})}$ sont des résumés ponctuels de chaque catégorie $\mathrm{C}_{\mathrm{j}}$ conditionnée par chaque $\mathbf{S}=\mathrm{s}_{\mathrm{i}}$, et on peut considérer que c'est l'espérance d'une variable aléatoire $\mathbf{B}_{\mathrm{j}}$ attachée à la catégorie, c'est-à-dire $c_{j(i)}=E\left(\mathbf{B}_{j} \mid \mathbf{S}=\mathrm{s}_{\mathrm{i}}\right)$. Pour un résumé unique sur l'ensemble des données on peut ainsi prendre $c_{j}=E_{S} E\left(\mathbf{B}_{j} \mid \mathbf{S}\right)=E\left(\mathbf{B}_{j}\right)=E_{S}\left(u_{i}+\sigma_{i} / \Delta F_{i j}\right.$ $\mathrm{K}_{\mathrm{i}, \mathrm{j}}$ ) en utilisant la relation de l'espérance totale.

Avec une loi discrète pour $\mathbf{S}$ cela donne la relation $\mathrm{c}_{\mathrm{j}}=\sum_{\mathrm{i}} \frac{\mathrm{n}_{\mathrm{i}+}}{\mathrm{n}} \mathrm{c}_{\mathrm{j}(\mathrm{i})}=\sum_{\mathrm{i}} \frac{\mathrm{n}_{\mathrm{i}+}}{\mathrm{n}} \mathrm{u}_{\mathrm{i}}$ $+\sum_{\mathrm{i}} \frac{\mathrm{n}_{\mathrm{i}+}}{\mathrm{n}} \frac{\mathrm{K}_{\mathrm{i}, \mathrm{j}} \sigma_{i}}{\Delta \mathrm{F}_{\mathrm{ij}}}$ avec le terme $\sum_{\mathrm{i}} \frac{\mathrm{n}_{\mathrm{i}+}}{\mathrm{n}} \mathrm{u}_{\mathrm{i}}=\mathrm{E}_{\mathrm{S}} \mathrm{E}\left(\mathbf{U} \mid \mathbf{S}=\mathrm{s}_{\mathrm{i}}\right)=\mathrm{E}(\mathbf{U})$ commun à tout les $\mathrm{c}_{\mathrm{j}}$, et un terme $\sum_{\mathrm{i}} \frac{\mathrm{n}_{\mathrm{i}+}}{\mathrm{n}} \frac{\mathrm{K}_{\mathrm{i}, \mathrm{j}} \sigma_{i}}{\Delta \mathrm{F}_{\mathrm{ij}}}$ spécifique à chaque catégorie.

II - Le calcul des $c_{\mathrm{j}}$ repose sur les intégrales $\mathrm{K}_{\mathrm{i}, \mathrm{j}}$, ci-dessous nous explicitons le calcul des primitives $\mathrm{K}(\mathrm{x})=\int \mathrm{x} f(\mathrm{x})$ dx pour les fonctions de répartition $\mathrm{F}$ retenues dans le texte.

\section{II.1 - LOI NORMALE}

On a $f(x)=\frac{e^{-x^{2} / 2}}{\sqrt{2 \pi}}$ et donc $K(x)=\int x \frac{e^{-x^{2} / 2}}{\sqrt{2 \pi}} d x$. 
En prenant le changement de variable $v=x^{2} / 2$ on a $K(x)=\int \frac{e^{-v}}{\sqrt{2 \pi}} d v=-\frac{e^{-v}}{\sqrt{2 \pi}}$ c'est-à-dire $K(x)=-\frac{e^{-x^{2} / 2}}{\sqrt{2 \pi}}$, une application paire qui s'annule pour $\pm \infty$.

\section{2 - LOI LOGISTIQUE}

On a $F(x)=\frac{1}{1+e^{-x}}$ et $K(x)=\int x d\left(\frac{1}{1+e^{-x}}\right)=\frac{x}{1+e^{-x}}+H(x)$ avec $H(x)=-\int \frac{d x}{1+e^{-x}}$ en intégrant par parties. On a encore $H=\int \frac{d u}{u(1+u)}$ avec le changement de variable $\mathrm{u}=\mathrm{e}^{-\mathrm{x}}$, à savoir $\mathrm{H}=\int \frac{\mathrm{du}}{\mathrm{u}}-\int \frac{\mathrm{du}}{1+\mathrm{u}}=\ln \mathrm{u}-\ln (1+\mathrm{u})$, ce qui donne $\mathrm{H}(\mathrm{x})=-\mathrm{x}-\ln \left(1+\mathrm{e}^{-\mathrm{x}}\right)$.

Il en résulte que $K(x)=\frac{-x}{1+e^{x}}-\ln \left(1+e^{-x}\right)=x \frac{e^{x / 2}-e^{-x / 2}}{2\left(e^{x / 2}+e^{-x / 2}\right)}-\ln \left(e^{x / 2}+e^{-x / 2}\right)$, une application paire qui s'annule pour $\pm \infty$.

\section{II.3 - LOI DES VALEURS EXTRÊMES DE FRÉCHET FISHER TIPPETT :}

On a $\mathrm{F}(\mathrm{x})=\exp \left(-\mathrm{e}^{-\mathrm{x}}\right)$ et, à la différence des précédentes, cette distribution est non symétrique. Ici nous prenons la primitive $\mathrm{K}(\mathrm{x})=\int_{0}^{\mathrm{x}} \mathrm{u} \mathrm{dF}(\mathrm{u})$ qui s'annule à l'origine.

a) pour $x>0$

on a $\mathrm{K}(\mathrm{x})=\int_{0}^{\mathrm{x}} \mathrm{u} \mathrm{d}(\mathrm{F}(\mathrm{u})-1)=\mathrm{x}(\mathrm{F}(\mathrm{x})-1)+\mathrm{J}(\mathrm{x})$ avec $\mathrm{J}(\mathrm{x})=\int_{0}^{\mathrm{x}}(1-\mathrm{F}(\mathrm{u}))$ du après une intégration par parties. On a encore $\mathrm{J}(\mathrm{x})=\int_{\mathrm{e}^{-\mathrm{x}}}{ }^{1}\left(1-\mathrm{e}^{-\mathrm{t}}\right) / \mathrm{t} \mathrm{dt}$ avec le changement de variable $\mathrm{t}=\mathrm{e}^{-\mathrm{u}}$.

Cette dernière intégrale est connue, en effet $\varphi(v)=\int_{0}^{v}\left(1-e^{-t}\right) / t d t$ est égal à $E_{1}(v)+\ln$ $v+\gamma$ avec $\gamma$ la constante d'Euler, et $E_{1}(x)=\int_{x}^{\infty} e^{-v} / v d v$ une fonction liée au logarithme intégral [Abramowitz, Stegun, 1970].

On en déduit $K(x)=x(F(x)-1)+\varphi(1)-\varphi\left(e^{-x}\right)$

ou encore $\mathrm{x}(\mathrm{F}(\mathrm{x})-1)+\mathrm{E}_{1}(1)-\mathrm{E}_{1}\left(\mathrm{e}^{-\mathrm{x}}\right)+\mathrm{x}=\mathrm{x} \exp \left(-\mathrm{e}^{-\mathrm{x}}\right)+\mathrm{E}_{1}(1)-\mathrm{E}_{1}\left(\mathrm{e}^{-\mathrm{x}}\right)$. Quand $\mathrm{x}$ tend vers plus l'infini $x(F(x)-1)$ tend vers zéro, par ailleurs $\varphi(u)$ tend vers zéro quand $u$ tend vers zéro, il en résulte que $K(x)$ tend vers $\varphi(1)=E_{1}(1)+\gamma$ quand $x$ tend vers plus l'infini.

b) pour $\mathrm{x}<0$

$\mathrm{K}(\mathrm{x})=\int_{0}^{\mathrm{x}} \mathrm{u} \mathrm{dF}(\mathrm{u})=\mathrm{x} \mathrm{F}(\mathrm{x})+\mathrm{L}(\mathrm{x})$ avec $\mathrm{L}(\mathrm{x})=-\int_{0}^{\mathrm{x}} \mathrm{F}(\mathrm{u}) \mathrm{du}=\int_{1}^{\mathrm{e}^{-\mathrm{x}}} \mathrm{e}^{-\mathrm{t} / \mathrm{t}} \mathrm{dt}=\mathrm{E}_{1}(1)-$ $E_{1}\left(e^{-x}\right)$, il en résulte que l'on a encore $K(x)=x \exp \left(-e^{-x}\right)+E_{1}(1)-E_{1}\left(e^{-x}\right)$. Quand $x$ tend vers moins l'infini $x \mathrm{~F}(\mathrm{x})$ tend vers zéro, et $\mathrm{E}_{1}(\mathrm{u})$ tend vers zéro quand $\mathrm{u}$ tend plus l'infini, il en résulte que $\mathrm{K}(\mathrm{x})$ tend vers $\mathrm{E}_{1}(1)$ quand $\mathrm{x}$ tend vers moins l'infini. 
De bonnes approximations de $\mathrm{E}_{1}$ figurent dans [Abramowitz, Stegun, 1970], K(x) est ainsi connue pour tout $\mathrm{x}$, avec $\mathrm{K}(\infty)=\mathrm{E}_{1}(1)+\gamma$ et $\mathrm{K}(-\infty)=\mathrm{E}_{1}(1)=0,2193839 \ldots$

\section{II.4 - LOI DES VALEURS EXTRÊMES DE FRÉCHET FISHER TIPPETT CHANGÉE DE SIGNE}

C'est une autre loi non symétrique sur $\mathrm{R}$ qui a pour fonction de répartition $\mathrm{F}_{\mathrm{FFT}-}(\mathrm{x})=1-\mathrm{F}_{\mathrm{FFT}}(-\mathrm{x})$, par conséquent $\mathrm{K}_{\mathrm{FFT}-}(\mathrm{x})=-\mathrm{K}_{\mathrm{FFT}}(-\mathrm{x})$ est connue à partir du $\mathrm{K}_{\mathrm{FFT}}$ en II.3.

\section{ANNEXE 2 \\ DES QUANTILES EN GUISE DE PSEUDO BORNES EXTREMMALES POUR $\mathrm{T}_{0}$ ET $\mathrm{T}_{\mathrm{J}}$}

Par construction les bornes extrémales des deux catégories en extrémités de l'échelle des réponses $C_{1}$ et $C_{J}$ sont infinies, $t_{0}=-\infty$ et $t_{J}=\infty$. Cependant on pourrait aimer disposer d'un ordre de grandeur de valeurs extrémales finies pour l'échelle de mesurage que l'on obtient. Ceci est rendu possible grâce à la nature probabiliste des intervalles successifs et à la notion de quantile.

On rappelle que $\mathrm{P}\left(\mathbf{U}_{\mathrm{i}} \leq \mathrm{t}\right)=\mathrm{P}\left(\mathbf{U} \leq \mathrm{t} \mid \mathbf{S}=\mathrm{s}_{\mathrm{i}}\right)=\mathrm{F}_{\mathrm{i}}(\mathrm{t})=\mathrm{F}\left(\frac{\mathrm{t}-\mathrm{u}_{\mathrm{i}}}{\sigma_{\mathrm{i}}}\right)$, et avec la relation de l'espérance totale $\mathrm{P}(\mathbf{U} \leq \mathrm{t})=\mathrm{E}_{\mathrm{S}}(\mathrm{P}(\mathbf{U} \leq \mathrm{t} \mid \mathbf{S}))$ on introduit le quantile établi sur l'ensemble des données en posant la définition $\mathrm{P}\left(\mathbf{U} \leq \mathrm{t}_{\mathrm{q}}\right)=\mathrm{E}_{\mathrm{S}}\left(\mathrm{P}\left(\mathbf{U} \leq \mathrm{t}_{\mathrm{q}} \mathbf{S}\right)\right)=\mathrm{E}_{\mathrm{S}}\left(\mathrm{F}\left(\frac{\mathrm{t}_{\mathrm{q}}-\mathrm{u}_{\mathrm{i}}}{\sigma_{\mathrm{i}}}\right)\right)=\mathrm{q}$.

Avec une distribution discrète $\mathrm{t}_{\mathrm{q}}$ est la racine de l'équation $\sum_{\mathrm{i}} \frac{\mathrm{n}_{\mathrm{i}+}}{\mathrm{n}} \mathrm{F}\left(\frac{\mathrm{t}-\mathrm{u}_{\mathrm{i}}}{\sigma_{\mathrm{i}}}\right)-\mathrm{q}=0$. Le premier membre est croissant en $\mathrm{t}$, négatif pour $\inf \left(\mathrm{t}_{\mathrm{q}(\mathrm{i})}\right)$ et positif pour $\sup \left(\mathrm{t}_{\mathrm{q}(\mathrm{i})}\right)$, en posant $\mathrm{t}_{\mathrm{q}(\mathrm{i})}$ le quantile des lois conditionnelles. Il en résulte qu'il existe une racine unique à l'équation en $\mathrm{t}$ pour toute probabilité $\mathrm{q}$, et que l'on peut ainsi envisager de prendre par exemple les quantiles $t_{0,01}$ pour $t_{0}$ et $t_{0,99}$ pour $t_{J}$ au titre de pseudo bornes extrémales. 


\section{ANNEXE 3 \\ QUELQUES RAPPELS À PROPOS DU CONDITIONNEMENT PAR DES VARIABLES ALÉATOIRES}

La nouvelle formulation des intervalles successifs au $\S 4.2$ dans le texte fait appel au conditionnement par des variables aléatoires et à des prises d'espérance sous ce conditionnement.

I - Avec les variables aléatoires $\mathbf{Y}$ et $\mathbf{S}$, l'espérance conditionnelle $\mathrm{E}(\mathbf{Y} \mid \mathbf{S})$, (prise selon la loi de $\mathbf{Y}$ ), est une nouvelle variable aléatoire transformée de $\mathbf{S}$, et son espérance $\mathrm{E}_{\mathbf{S}}(\mathrm{E}(\mathbf{Y} \mid \mathbf{S}))$ est égale à $\mathrm{E}(\mathbf{Y})$ moyennant la relation de l'espérance totale.

Avec deux variables de conditionnement $\mathbf{S}_{1}$ et $\mathbf{S}_{2}$, l'espérance $\mathrm{E}\left(\mathbf{Y} \mid \mathbf{S}_{1}, \mathbf{S}_{2}\right)$, (prise selon la loi de $\mathbf{Y})$, est une variable aléatoire $\mathrm{g}\left(\mathbf{S}_{1}, \mathbf{S}_{2}\right)$ transformée de $\mathbf{S}_{1}$ et $\mathbf{S}_{2}$. En tant que telle, elle possède des espérances partielles avec une seule variable de conditionnement. Par exemple l'espérance $\mathrm{E}_{\mathrm{S} 2}\left(\mathrm{~g}\left(\mathbf{S}_{1}, \mathbf{S}_{2}\right) \mid \mathbf{S}_{1}\right)$ prise en fonction de la seule loi de $\mathbf{S}_{2}$ est une transformée $\mathrm{g}_{1}\left(\mathbf{S}_{1}\right)$ de la seule variable $\mathbf{S}_{1}$, et dans le cas présent il s'agit de $\mathrm{E}_{\mathrm{S} 2}\left(\mathrm{E}\left(\mathbf{Y} \mid \mathbf{S}_{1}, \mathbf{S}_{2}\right) \mid \mathbf{S}_{1}\right)$. La généralisation de la relation de l'espérance totale avec une variable de conditionnement nous indique que $\mathrm{E}_{\mathrm{S} 2}\left(\mathrm{E}\left(\mathbf{Y} \mid \mathbf{S}_{1}, \mathbf{S}_{2}\right) \mid \mathbf{S}_{1}\right)$ est égale à $\mathrm{E}\left(\mathbf{Y} \mid \mathbf{S}_{1}\right)$ [Feller, 1971] (au besoin on vérifie bien que les deux expressions sont des transformées de la seule $\mathbf{S}_{1}$ ). En dernier les espérances en $\mathbf{S}_{1}$ de ces deux quantités égales donnent $\mathrm{E}_{\mathrm{S} 1}\left\{\mathrm{E}_{\mathrm{S} 2}\left(\mathrm{E}\left(\mathbf{Y} \mid \mathbf{S}_{1}, \mathbf{S}_{2}\right) \mid \mathbf{S}_{1}\right)\right\}=\mathrm{E}_{\mathrm{S} 1}\left(\mathrm{E}\left(\mathbf{Y} \mid \mathbf{S}_{1}\right)\right)=\mathrm{E}(\mathbf{Y})$. Naturellement on a les relations analogues $\mathrm{E}_{\mathrm{S} 1}\left(\mathrm{E}\left(\mathbf{Y} \mid \mathbf{S}_{1}, \mathbf{S}_{2}\right) \mid \mathbf{S}_{2}\right)=\mathrm{E}\left(\mathbf{Y} \mid \mathbf{S}_{2}\right)$ et $\mathrm{E}_{\mathrm{S} 2}\left\{\mathrm{E}_{\mathrm{S} 1}\left(\mathrm{E}\left(\mathbf{Y} \mid \mathbf{S}_{1}, \mathbf{S}_{2}\right) \mid \mathbf{S}_{2}\right)\right\}=$ $\mathrm{E}_{\mathrm{S} 2}\left(\mathrm{E}\left(\mathbf{Y} \mid \mathbf{S}_{2}\right)\right)=\mathrm{E}(\mathbf{Y})$ en permutant les variables.

NOTE : Dans ces calculs l'indice ${ }_{\mathrm{S}}$ ou $\mathrm{Si}_{\mathrm{i}} \mathrm{i}=1,2$ désigne l'espérance par rapport à la loi de $\mathbf{S}$ ou de $\mathbf{S}_{\mathrm{i}}$, et on ne fait pas figurer l'indice y pour l'espérance par rapport à la loi de $\mathbf{Y}$.

II - Les espérances précédentes sont prises en toute généralité avec la, ou les, variable(s) $\mathbf{S}$ ou $\mathbf{S}_{\mathrm{i}}$ de conditionnement, auquel cas $\mathrm{E}(\mathbf{Y} \mid \mathbf{S})$ et $\mathrm{E}\left(\mathbf{Y} \mid \mathbf{S}_{1}, \mathbf{S}_{2}\right)$ sont des transformées aléatoires.

Mais on a aussi la situation où la (les) variable(s) de conditionnement a (ont) une valeur donnée s ou $\mathrm{s}_{\mathrm{i}}$. Dans ce cas $\mathrm{E}(\mathbf{Y} \mid \mathbf{S}=\mathrm{s})$ est un nombre, $\mathrm{E}\left(\mathbf{Y} \mid \mathbf{S}_{1}=\mathrm{s}_{1}, \mathbf{S}_{2}=\mathrm{s}_{2}\right)$ aussi, tandis que $\mathrm{E}\left(\mathbf{Y} \mid \mathbf{S}_{1}=\mathrm{s}_{1}, \mathbf{S}_{2}\right)$ est une variable aléatoire transformée de la seule $\mathbf{S}_{2}$ et que $\mathrm{E}\left(\mathbf{Y} \mid \mathbf{S}_{1}, \mathbf{S}_{2}=\mathrm{s}_{2}\right)$ est une transformée de la seule $\mathbf{S}_{1}$.

Toutes ces situations se présentent dans les calculs du texte. 


\section{ANNEXE 4 \\ LA VARIANCE DE E( $\left(\mathbf{Y} \mid \mathbf{S}_{1}, \mathbf{S}_{2}\right)$}

\section{LES MOMENTS DE $\mathbf{Z}=\mathrm{g}\left(\mathbf{S}_{1}, \mathbf{S}_{2}\right)$}

En premier on considère la variable aléatoire générale $\mathbf{Z}$, transformée de $\mathbf{S}_{1}$ et $\mathbf{S}_{2}$.

a) calcul de l'espérance : $\mathrm{E}(\mathbf{Z})=\mathrm{E}_{\mathrm{S} 1}\left[\mathrm{E}_{\mathrm{S} 2}\left(\mathrm{~g}\left(\mathbf{S}_{1}, \mathbf{S}_{2}\right) \mid \mathbf{S}_{1}\right)\right]=\mathrm{E}_{\mathrm{S} 2}\left[\mathrm{E}_{\mathrm{S} 1}\left(\mathrm{~g}\left(\mathbf{S}_{1}, \mathbf{S}_{2}\right) \mid \mathbf{S}_{2}\right)\right]$;

b) calcul de la variance : $\operatorname{var}(\mathbf{Z})=\mathrm{E}(\mathbf{Z}-\mathrm{E}(\mathbf{Z}))^{2}=\mathrm{E}_{\mathrm{S} 1}\left[\mathrm{E}_{\mathrm{S} 2}\left\{[\mathbf{Z}-\mathrm{E}(\mathbf{Z})]^{2} \mid \mathbf{S}_{1}\right\}\right]$

$$
\begin{aligned}
& =\mathrm{E}_{\mathrm{S} 1}\left[\mathrm{E}_{\mathrm{S} 2}\left\{\left[\mathrm{~g}\left(\mathbf{S}_{1}, \mathbf{S}_{2}\right)-\mathrm{E}\left(\mathrm{g}\left(\mathbf{S}_{1}, \mathbf{S}_{2}\right)\right)\right]^{2} \mid \mathbf{S}_{1}\right\}\right] \\
& =\mathrm{E}_{\mathrm{S} 1}\left[\mathrm{E}_{\mathrm{S} 2}\left\{\left[\mathrm{~g}\left(\mathbf{S}_{1}, \mathbf{S}_{2}\right)-\mathrm{E}_{\mathrm{S} 2}\left(\mathrm{~g}\left(\mathbf{S}_{1}, \mathbf{S}_{2}\right) \mid \mathbf{S}_{1}\right)+\mathrm{E}_{\mathrm{S} 2}\left(\mathrm{~g}\left(\mathbf{S}_{1}, \mathbf{S}_{2}\right) \mid \mathbf{S}_{1}\right)-\mathrm{E}\left(\mathrm{g}\left(\mathbf{S}_{1}, \mathbf{S}_{2}\right)\right)\right]^{2} \mid \mathbf{S}_{1}\right\}\right] \\
& =\mathrm{E}_{\mathrm{S} 1}\left[\mathrm{E}_{\mathrm{S} 2}\left\{\left[\mathrm{~g}\left(\mathbf{S}_{1}, \mathbf{S}_{2}\right)-\mathrm{E}_{\mathrm{S} 2}\left(\mathrm{~g}\left(\mathbf{S}_{1}, \mathbf{S}_{2}\right) \mid \mathbf{S}_{1}\right)\right]^{2} \mid \mathbf{S}_{1}\right\}\right] \\
& +\mathrm{E}_{\mathrm{S} 1}\left[\mathrm{E}_{\mathrm{S} 2}\left\{\left[\mathrm{E}_{\mathrm{S} 2}\left(\mathrm{~g}\left(\mathbf{S}_{1}, \mathbf{S}_{2}\right) \mid \mathbf{S}_{1}\right)-\mathrm{E}\left(\mathrm{g}\left(\mathbf{S}_{1}, \mathbf{S}_{2}\right)\right)\right]^{2} \mid \mathbf{S}_{1}\right\}\right] \\
& +2 \mathrm{E}_{\mathrm{S} 1}\left[\mathrm{E}_{\mathrm{S} 2}\left\{\left[\mathrm{~g}\left(\mathbf{S}_{1}, \mathbf{S}_{2}\right)-\mathrm{E}_{\mathrm{S} 2}\left(\mathrm{~g}\left(\mathbf{S}_{1}, \mathbf{S}_{2}\right) \mid \mathbf{S}_{1}\right)\right] \cdot\left[\mathrm{E}_{\mathrm{S} 2}\left(\mathrm{~g}\left(\mathbf{S}_{1}, \mathbf{S}_{2}\right) \mid \mathbf{S}_{1}\right)-\mathrm{E}\left(\mathrm{g}\left(\mathbf{S}_{1}, \mathbf{S}_{2}\right)\right)\right] \mid \mathbf{S}_{1}\right\}\right] .
\end{aligned}
$$

- Dans le premier terme $\mathrm{E}_{\mathrm{S} 2}\left\{\left[\mathrm{~g}\left(\mathbf{S}_{1}, \mathbf{S}_{2}\right)-\mathrm{E}_{\mathrm{S} 2}\left(\mathrm{~g}\left(\mathbf{S}_{1}, \mathbf{S}_{2}\right) \mid \mathbf{S}_{1}\right)\right]^{2} \mid \mathbf{S}_{1}\right\}=\operatorname{var}_{\mathrm{S} 2}\left(\mathrm{~g}\left(\mathbf{S}_{1}, \mathbf{S}_{2}\right) \mid \mathbf{S}_{1}\right)$, et le premier terme est donc égal à $\mathrm{E}_{\mathrm{S} 1}\left[\operatorname{var}_{\mathrm{S} 2}\left(\mathrm{~g}\left(\mathbf{S}_{1}, \mathbf{S}_{2}\right) \mid \mathbf{S}_{1}\right)\right]$.

- Dans le second, $\mathrm{E}_{\mathrm{S} 2}\left\{\left[\mathrm{E}_{\mathrm{S} 2}\left(\mathrm{~g}\left(\mathbf{S}_{1}, \mathbf{S}_{2}\right) \mid \mathbf{S}_{1}\right)-\mathrm{E}\left(\mathrm{g}\left(\mathbf{S}_{1}, \mathbf{S}_{2}\right)\right)\right]^{2} \mid \mathbf{S}_{1}\right\}$ ne dépend pas de $\mathbf{S}_{2}$, cette quantité est donc égale à

$$
\left[\mathrm{E}_{\mathrm{S} 2}\left(\mathrm{~g}\left(\mathbf{S}_{1}, \mathbf{S}_{2}\right) \mid \mathbf{S}_{1}\right)-\mathrm{E}\left(\mathrm{g}\left(\mathbf{S}_{1}, \mathbf{S}_{2}\right)\right)\right]^{2}=\left[\mathrm{E}_{\mathrm{S} 2}\left(\mathrm{~g}\left(\mathbf{S}_{1}, \mathbf{S}_{2}\right) \mid \mathbf{S}_{1}\right)-\mathrm{E}_{\mathrm{S} 1}\left[\mathrm{E}_{\mathrm{S} 2}\left(\mathrm{~g}\left(\mathbf{S}_{1}, \mathbf{S}_{2}\right) \mid \mathbf{S}_{1}\right)\right]\right]^{2},
$$

et le deuxième terme est égal à $\operatorname{var}_{\mathrm{S} 1}\left[\mathrm{E}_{\mathrm{S} 2}\left(\mathrm{~g}\left(\mathbf{S}_{1}, \mathbf{S}_{2}\right) \mid \mathbf{S}_{1}\right)\right]$.

- Dans le troisième terme on a

$$
\begin{aligned}
& \mathrm{E}_{\mathrm{S} 2}\left\{\left[\mathrm{~g}\left(\mathbf{S}_{1}, \mathbf{S}_{2}\right)-\mathrm{E}_{\mathrm{S} 2}\left(\mathrm{~g}\left(\mathbf{S}_{1}, \mathbf{S}_{2}\right) \mid \mathbf{S}_{1}\right)\right] \cdot\left[\mathrm{E}_{\mathrm{S} 2}\left(\mathrm{~g}\left(\mathbf{S}_{1}, \mathbf{S}_{2}\right) \mid \mathbf{S}_{1}\right)-\mathrm{E}\left(\mathrm{g}\left(\mathbf{S}_{1}, \mathbf{S}_{2}\right)\right)\right] \mid \mathbf{S}_{1}\right\}= \\
& {\left[\mathrm{E}_{\mathrm{S} 2}\left(\mathrm{~g}\left(\mathbf{S}_{1}, \mathbf{S}_{2}\right) \mid \mathbf{S}_{1}\right)-\mathrm{E}\left(\mathrm{g}\left(\mathbf{S}_{1}, \mathbf{S}_{2}\right)\right)\right] . \mathrm{E}_{\mathrm{S} 2}\left\{\left[\mathrm{~g}\left(\mathbf{S}_{1}, \mathbf{S}_{2}\right)-\mathrm{E}_{\mathrm{S} 2}\left(\mathrm{~g}\left(\mathbf{S}_{1}, \mathbf{S}_{2}\right) \mid \mathbf{S}_{1}\right)\right] \mid \mathbf{S}_{1}\right\}=0 ;}
\end{aligned}
$$

- il en résulte que

$$
\operatorname{var}\left(\mathrm{g}\left(\mathbf{S}_{1}, \mathbf{S}_{2}\right)\right)=\mathrm{E}_{\mathrm{S} 1}\left[\operatorname{var}_{\mathbf{S} 2}\left(\mathrm{~g}\left(\mathbf{S}_{1}, \mathbf{S}_{2}\right) \mid \mathbf{S}_{1}\right)\right]+\operatorname{var}_{\mathrm{S} 1}\left[\mathrm{E}_{\mathrm{S} 2}\left(\mathrm{~g}\left(\mathbf{S}_{1}, \mathbf{S}_{2}\right) \mid \mathbf{S}_{1}\right)\right]
$$

II. APPLICATION AU CAS $\mathrm{g}\left(\mathbf{S}_{1}, \mathbf{S}_{2}\right)=\mathrm{E}\left(\mathbf{Y} \mid \mathbf{S}_{1}, \mathbf{S}_{2}\right)$

a) dans ce cas on a la relation de cohérence entre les espérances conditionnelles et leurs espérances partielles $\mathrm{E}_{\mathrm{S} 2}\left(\mathrm{E}\left(\mathbf{Y} \mid \mathbf{S}_{1}, \mathbf{S}_{2}\right) \mid \mathbf{S}_{1}\right)=\mathrm{E}\left(\mathbf{Y} \mid \mathbf{S}_{1}\right)$,

b) on en déduit l'espérance $\mathrm{E}(\mathrm{g})=\mathrm{E}(\mathbf{Y})$ avec la relation de l'espérance totale,

c) pour la variance totale on a donc

$$
\operatorname{var}\left(\mathrm{E}\left(\mathbf{Y} \mid \mathbf{S}_{1}, \mathbf{S}_{2}\right)\right)=\mathrm{E}_{\mathrm{S} 1}\left[\operatorname{var}_{\mathrm{S} 2}\left(\mathrm{E}\left(\mathbf{Y} \mid \mathbf{S}_{1}, \mathbf{S}_{2}\right) \mid \mathbf{S}_{1}\right)\right]+\operatorname{var}_{\mathrm{S} 1}\left(\mathrm{E}\left(\mathbf{Y} \mid \mathbf{S}_{1}\right)\right)
$$


de même que $\mathrm{E}_{\mathrm{S} 2}\left[\operatorname{var}_{\mathrm{S} 1}\left(\mathrm{E}\left(\mathbf{Y} \mid \mathbf{S}_{1}, \mathbf{S}_{2}\right) \mid \mathbf{S}_{2}\right)\right]+\operatorname{var}_{\mathrm{S} 2}\left(\mathrm{E}\left(\mathbf{Y} \mid \mathbf{S}_{2}\right)\right)$ en permutant les variables.

En procédant en deux étapes on peut prendre les espérances conditionnelles dans deux ordres différents, et chacun d'eux rompt la symétrie a priori du rôle des variables. Lorsque les deux variables du plan d'expérience ont des rôles différents, l'un des deux développements de $\operatorname{var}\left(\mathrm{E}\left(\mathbf{Y} \mid \mathbf{S}_{1}, \mathbf{S}_{2}\right)\right)$ peut ainsi posséder une interprétation plus naturelle que l'autre. Ce calcul classique s'en tient à la seule dépendance en fonction de $\mathbf{S}_{1}$ et $\mathbf{S}_{2}$, alors que la décomposition du Théorème 1 fait intervenir les espérances conditionnelles $\mathrm{E}\left(\mathbf{Y} \mid \mathbf{S}_{1}\right)$ et $\mathrm{E}\left(\mathbf{Y} \mid \mathbf{S}_{2}\right)$.

ANNEXE 5

DES COMPLÉMENTS FORMELS SUR L'INTERACTION

DANS LE CAS GÉNÉRAL

Nous revenons sur le terme général $c_{\text {dep }}\left(\mathbf{S}_{1}, \mathbf{S}_{2}\right)=\mathrm{E}\left(\mathbf{U} \mid \mathbf{S}_{1}, \mathbf{S}_{2}\right)-\mathrm{a}_{1}\left(\mathbf{S}_{1}\right)-\mathrm{a}_{2}\left(\mathbf{S}_{2}\right)$ posé comme le complément à la solution $\mathrm{E}\left(\mathbf{U} \mid \mathbf{S}_{1}, \mathbf{S}_{2}\right)$ avec les applications $\mathrm{a}_{1}\left(\mathbf{S}_{1}\right)$ et $\mathrm{a}_{2}\left(\mathbf{S}_{2}\right)$ qui vérifient les conditions nécessaires du Théorème 3 . Sous réserve de l'existence de $\mathrm{a}_{1}$ et $\mathrm{a}_{2}$ ce coefficient possède quelques propriétés formelles.

LEMME A1 - Le terme d'interaction $\mathrm{c}_{\mathrm{dep}}$ est centré.

En effet les applications vérifient les conditions nécessaires du Théorème 3

$$
\begin{aligned}
& \mathrm{a}_{1}\left(\mathbf{S}_{1}\right)=\mathrm{E}\left(\mathbf{U} \mid \mathbf{S}_{1}\right)-\mathrm{E}_{\mathrm{S} 2}\left(\mathrm{a}_{2}\left(\mathbf{S}_{2}\right) \mid \mathbf{S}_{1}\right) \\
& \mathrm{a}_{2}\left(\mathbf{S}_{2}\right)=\mathrm{E}\left(\mathbf{U} \mid \mathbf{S}_{2}\right)-\mathrm{E}_{\mathrm{S} 1}\left(\mathrm{a}_{1}\left(\mathbf{S}_{1}\right) \mid \mathbf{S}_{2}\right),
\end{aligned}
$$

il en résulte que

$$
\mathrm{E}_{\mathrm{S} 1}\left(\mathrm{E}\left(\mathbf{U} \mid \mathbf{S}_{1}\right)\right)=\mathrm{E}_{\mathrm{S} 1}\left(\mathrm{a}_{1}\left(\mathbf{S}_{1}\right)\right)+\mathrm{E}_{\mathrm{S} 1}\left(\mathrm{E}_{\mathrm{S} 2}\left(\mathrm{a}_{2}\left(\mathbf{S}_{2}\right) \mid \mathbf{S}_{1}\right)\right)
$$

c'est-à-dire $\mathrm{E}(\mathbf{U})=\mathrm{E}_{\mathrm{S} 1}\left(\mathrm{a}_{1}\left(\mathbf{S}_{1}\right)\right)+\mathrm{E}_{\mathrm{S} 2}\left(\mathrm{E}_{\mathrm{S} 1}\left(\mathrm{a}_{2}\left(\mathbf{S}_{2}\right) \mid \mathbf{S}_{2}\right)\right)=\mathrm{E}_{\mathrm{S} 1}\left(\mathrm{a}_{1}\left(\mathbf{S}_{1}\right)\right)+\mathrm{E}_{\mathrm{S} 2}\left(\mathrm{a}_{2}\left(\mathbf{S}_{2}\right)\right)$.

LEMME A2 - Les espérances conditionnelles du terme $\mathrm{c}_{\mathrm{dep}}\left(\mathbf{S}_{1}, \mathbf{S}_{2}\right)$ sont identiquement nulles.

$$
\begin{aligned}
& \text { En effet on a } \mathrm{c}_{\mathrm{dep} 1}\left(\mathbf{S}_{1}\right)=\mathrm{E}_{\mathrm{S} 2}\left(\mathrm{c}_{\mathrm{dep}}\left(\mathbf{S}_{1}, \mathbf{S}_{2}\right) \mid \mathbf{S}_{1}\right) \\
& =\mathrm{E}_{\mathrm{S} 2}\left(\mathrm{E}\left(\mathbf{U} \mid \mathbf{S}_{1}, \mathbf{S}_{2}\right) \mid \mathbf{S}_{1}\right)-\mathrm{E}_{\mathrm{S} 2}\left(\mathrm{a}_{1}\left(\mathbf{S}_{1}\right) \mid \mathbf{S}_{1}\right)-\mathrm{E}_{\mathrm{S} 2}\left(\mathrm{E}\left(\mathrm{a}_{1}\left(\mathbf{S}_{2}\right) \mid \mathbf{S}_{1}\right)\right. \\
& =\mathrm{E}\left(\mathbf{U} \mid \mathbf{S}_{1}\right)-\mathrm{a}_{1}\left(\mathbf{S}_{1}\right)-\mathrm{E}_{\mathrm{S} 2}\left(\mathrm{E}\left(\mathrm{a}_{1}\left(\mathbf{S}_{2}\right) \mid \mathbf{S}_{1}\right)\right.
\end{aligned}
$$

qui est nul d'après les conditions nécessaires, et il en est de même pour

$$
\mathrm{c}_{\mathrm{dep} 2}\left(\mathbf{S}_{2}\right)=\mathrm{E}_{\mathrm{S} 1}\left(\mathrm{c}_{\mathrm{dep}}\left(\mathbf{S}_{1}, \mathbf{S}_{2}\right) \mid \mathbf{S}_{2}\right) .
$$

LEMME A3 - Dans le cadre des solutions d'une application des intervalles successifs, le terme d'interaction prend ses valeurs sur une échelle de rapport. 
Le système des conditions nécessaires pour $\mathrm{a}_{1}$ et $\mathrm{a}_{2}$ conduit aux deux relations (après élimination)

et $\begin{aligned} \mathrm{a}_{1}-\mathrm{E}_{\mathrm{S} 2}\left(\mathrm{E}_{\mathrm{S} 1}\left(\mathrm{a}_{1} \mid \mathbf{S}_{2}\right) \mid \mathbf{S}_{1}\right) & =\mathbf{U}_{1}-\mathrm{E}_{\mathrm{S} 2}\left(\mathbf{U}_{2} \mid \mathbf{S}_{1}\right) \\ \mathrm{a}_{2}-\mathrm{E}_{\mathrm{S} 1}\left(\mathrm{E}_{\mathrm{S} 2}\left(\mathrm{a}_{2} \mid \mathbf{S}_{1}\right) \mid \mathbf{S}_{2}\right) & =\mathbf{U}_{2}-\mathrm{E}_{\mathrm{S} 1}\left(\mathbf{U}_{1} \mid \mathbf{S}_{2}\right)\end{aligned}$

en posant pour simplifier $\mathbf{U}_{1}=\mathrm{E}\left(\mathbf{U} \mid \mathbf{S}_{1}\right)$ et $\mathbf{U}_{2}=\mathrm{E}\left(\mathbf{U} \mid \mathbf{S}_{2}\right)$ qui ne dépendent respectivement que de $\mathbf{S}_{1}$ et $\mathbf{S}_{2}$.

Cela montre que $a_{1}$ et $a_{2}$ varient linéairement en fonction des $\mathbf{U}_{r}, r=1,2$. Dans le cas des solutions des intervalles successifs, les $\mathbf{U}_{\mathrm{r}}$ prennent leurs valeurs sur une échelle d'intervalle, $\mathbf{U}_{1}-\mathrm{E}_{\mathrm{S} 2}\left(\mathbf{U}_{2} \mid \mathbf{S}_{1}\right)$ et $\mathbf{U}_{2}-\mathrm{E}_{\mathrm{S} 1}\left(\mathbf{U}_{1} \mid \mathbf{S}_{2}\right)$ aussi, et, par conséquent, les $\mathrm{a}_{1}$ et $\mathrm{a}_{2}$ solutions du système également (c'est la même échelle d'intervalle pour toutes ces valeurs).

En dernier

$$
\mathrm{c}_{\mathrm{dep}}\left(\mathbf{S}_{1}, \mathbf{S}_{2}\right)=\mathrm{E}\left(\mathbf{U} \mid \mathbf{S}_{1}, \mathbf{S}_{2}\right)-\mathrm{a}_{1}-\mathrm{a}_{2}=\mathrm{E}\left(\mathbf{U} \mid \mathbf{S}_{1}, \mathbf{S}_{2}\right)-\mathrm{E}(\mathbf{U})-\left\{\mathrm{a}_{1}-\mathrm{E}\left(\mathrm{a}_{1}\right)\right\}-\left\{\mathrm{a}_{2}-\mathrm{E}\left(\mathrm{a}_{2}\right)\right\},
$$

c'est une somme algébrique de trois variables centrées qui prennent leur valeur sur une échelle de rapport et leur différence également (avec la même constante multiplicative que celle de l'échelle d'intervalle de départ). 